FERMILAB-TM-2219 August 2003

\title{
COLLECTIVE INSTABILITIES
}

\author{
K.Y. Ng \\ Fermi National Accelerator Laboratory, P.O. Box 500, Batavia, IL 60510 \\ (June, 2003)
}

\begin{abstract}
The lecture covers mainly Sections 2.VIII and 3.VII of the book Accelerator Physics by S.Y. Lee, plus mode-coupling instabilities and chromaticity-driven head-tail instability. Besides giving more detailed derivation of many equations, simple interpretations of many collective instabilities are included with the intention that the phenomena can be understood more easily without going into too much mathematics. The notations of Lee's book as well as the $e^{j \omega t}$ convention aro followed.
\end{abstract}

Five-hour lecture given in part of the course

"Accelerator Physics"

US Particle Accelerator School

Santa Barbara, CA

June 16-27, 2003 


\section{WAKE FUNCTIONS}

A whole beam or a whole bunch can oscillate in many modes, like oscillating rigidly about the center of the beam pipe, breathing radially at a certain frequency with the center not moving at all, the horizontal breathing $90^{\circ}$ out of phase with the vertical breathing, etc. These are eigenmodes in an eigenfrequency, in which every particle in the beam is participating. We call these collective modes. If the amplitude of oscillation continues to grow, the beam becomes unstable. These modes are excited and driven by the discontinuities of the beam pipe or mechanically by kicker, etc.

A beam particle traveling along a "smooth" and perfectly conducting cylindrical beam pipe will have its pancake shape electromagnetic fields truncated by the beam pipe.* If the beam pipe is not smooth, however, electromagnetic fields will be trapped and experienced by beam particles following. These are called wakefields.

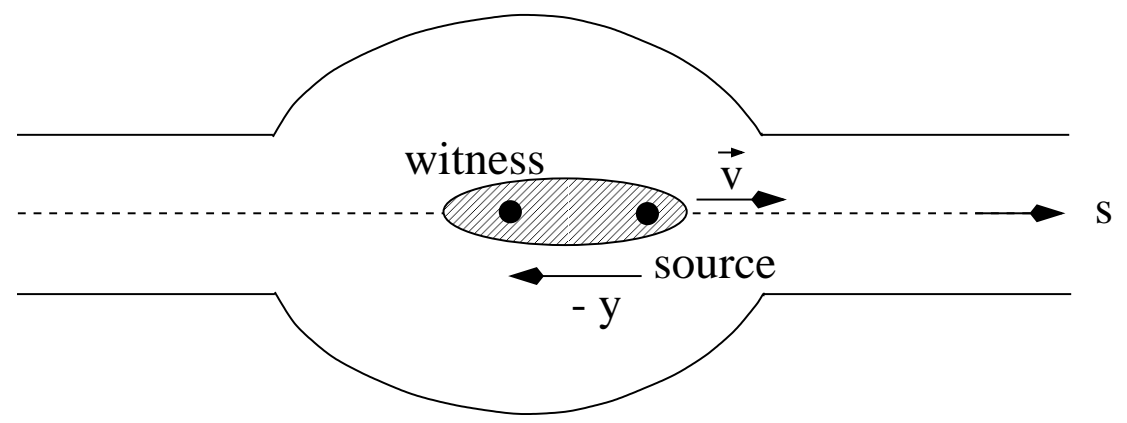

Figure 1: Schematic drawing of of a witness particle at a distance $-y$ behind the source particle in a beam. Both particles are traveling along the $s$ direction with the same longitudinal velocity $\vec{v}$.

We make two approximations:

(1) Rigid bunch approximation: the wakefields do not affect the rigidity of the beam during its transversal of the discontinuity. What we mean is that the bunch motion will not be affected during its passage and the effect of the wake is considered to be a kick after the passage.

(2) Impulse approximation: we care about only the impulse

$$
\Delta \vec{p}=\int_{-\infty}^{\infty} d t \vec{F}=\int_{-\infty}^{\infty} d t q(\vec{E}+\vec{v} \times \vec{B})
$$

impacted on the witness particle carrying charge $q$ traveling at longitudinal velocity $v$ during the discontinuity transversal, but not the force $\vec{F}$ nor separately the electric field $\vec{E}$ and magnetic flux density $\vec{B}$.

Besides the transverse positions of the source and witness particles, the impulse depends only on the longitudinal distance $y$ between them. Panofsky-Wenzel theorem [1] gives the

${ }^{*}$ Not simply truncation for a non-cylindrical beam pipe or for an off-axis beam. 
relation between the longitudinal component of the impulse and the transverse component: ${ }^{\dagger}$

$$
\frac{\partial}{\partial y} \Delta \vec{p}_{\perp}=\vec{\nabla}_{\perp} \Delta p_{s}
$$

For a cylindrically symmetric vacuum chamber, a source particle at an offset $a$ from the axis of the beam pipe can be expanded into cylindrical harmonics, and such an expansion is also valid for the impulse. We have

$$
\left\{\begin{array}{l}
v \Delta \vec{p}_{\perp}=-q I_{m} W_{m}(y) m r^{m-1}(\hat{r} \cos m \theta-\hat{\theta} \sin m \theta), \\
v \Delta p_{s}=-q I_{m} W_{m}^{\prime}(y) r^{m} \cos m \theta
\end{array}\right.
$$

where $(r, \theta)$ are the transverse coordinates of the witness particle carrying charge $q$. The source particle carrying charge $e$ is at coordinates $(a, 0)$ and is traveling in the $s$-direction. ${ }^{\ddagger}$ Its $m$ th electric moment is represented by $I_{m}=e a^{m}$. Here, $W_{m}(y)$ is called the wake function $^{\S}$ of the $m$ th azimuthal harmonic and is a function of $y$ only. Sometimes it is also expressed as a function of $t=-v / y$, the time lag of the witness particle. Strictly speaking, Eq. (1.3) is valid only at the ultra-relativistic limit or when the particle velocity $v \rightarrow c$. The longitudinal wake $W_{m}^{\prime}(y)=d W_{m}(y) / d y$ has the dimension volts/coulomb $/ \mathrm{m}^{2 m}$ while the transverse wake has the dimension volts/coulomb $/ \mathrm{m}^{2 m-1}$. Schematic drawings of $W_{m}(y)$ and $W_{m}^{\prime}(y)$ are shown in Fig. 2.
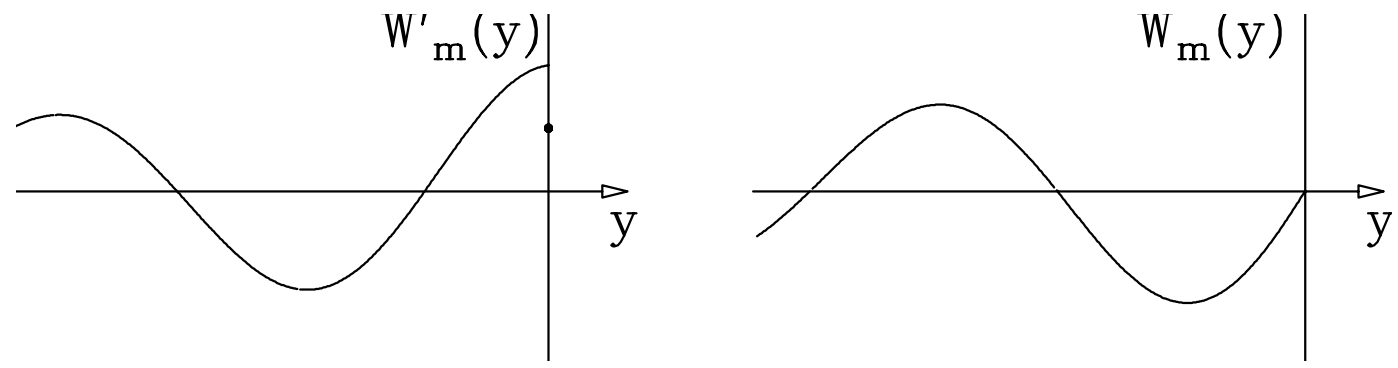

Figure 2: The longitudinal wake $W_{m}^{\prime}(y)$ is positive definite when $|y|$ is small, while the transverse wake $W_{m}(y)$ starts out from zero and goes negative as $|y|$ increases. They behave like cosine and sine, respectively, and vanish when $y>0$ because of causality.

\footnotetext{
${ }^{\dagger}$ Here, $y$ is measured from the source particle in the forward direction. Thus, when the witness particle is behind the witness particle, $y \leq 0$. On the other hand, if one prefers to define $y \geq 0$ as the distance the witness particle is behind the source particle, there will be a negative sign before the partial derivative in Eq. (1.2), and one needs to define $W_{m}^{\prime}(y) \equiv-d W_{m}(y) / d y$ in Eq. (1.3) below.

${ }^{\ddagger}$ Here, we follow the notation convention of $x$ and $z$ denoting, respectively, horizontal and vertical offsets from the closed orbit, while $y$ measuring the distance the witness particle ahead of the source particle, both traveling in the $s$-direction.

$\S$ Wake function is a Green's function because the source is a $\delta$-function.
} 


\section{COUPLING IMPEDANCES}

Beam particles form a current, of which the component with frequency $\omega /(2 \pi)$ is $I(s, t)=$ $\hat{I} e^{j \omega(t-s / v)}$, where $\hat{I}$ may be complex. This current component at location $s$ and time $t$ will be affected by the wake of the preceding beam particles at $s^{\prime}$ that pass the point $s$ at time $t-\left(s^{\prime}-s\right) / v$ with the charge element $I\left[s, t-\left(s^{\prime}-s\right) / v\right] d s^{\prime} / v$. The total accelerating voltage seen (or energy gained per unit test charge) will be

$$
\begin{aligned}
V(s, t) & =-\int_{s}^{\infty} I\left(s, t-\frac{s^{\prime}-s}{v}\right) W_{0}^{\prime}\left(s-s^{\prime}\right) \frac{d s^{\prime}}{v} \\
& =-\int_{-\infty}^{0} I\left(s, t+\frac{y}{v}\right) W_{0}^{\prime}(y) \frac{d y}{v} \\
& =-\int_{-\infty}^{\infty} \hat{I} e^{j \omega[t+y / v-s / v]} W_{0}^{\prime}(y) \frac{d y}{v} \\
& =-I(s, t) \int_{-\infty}^{\infty} e^{j \omega y / v} W_{0}^{\prime}(y) \frac{d y}{v}
\end{aligned}
$$

Thus, we can identify the longitudinal coupling impedance of the vacuum chamber as

$$
Z_{0}^{\|}(\omega)=\int_{-\infty}^{\infty} e^{j \omega y / v} W_{0}^{\prime}(y) \frac{d y}{v}
$$

This definition is the same as the ordinary impedance in a circuit. However, we have here much more than in a circuit because the current distribution possesses higher azimuthal multiples. In the same way, we define the transverse impedance

$$
Z_{1}^{\perp}(\omega)=\frac{-j}{\beta} \int_{-\infty}^{\infty} e^{j \omega y / v} W_{1}(z) \frac{d y}{v} .
$$

In Eqs. (2.1) and (2.3), the lower limits of integration have been extended to $-\infty$, because the wake functions vanish when $y>0$. It is evident that we can also compute the impedances by integrating the longitudinal wake force $F_{0}^{\|}$or transverse wake force $F_{1}^{\perp}$ at the frequency $\omega$ over one turn according to

$$
Z_{0}^{\|}(\omega)=-\frac{1}{q I_{0}} \int_{0}^{C} F_{0}^{\|}(s, t) d s, \quad Z_{1}^{\perp}(\omega)=\frac{j}{q \beta I_{0} a} \int_{0}^{C} F_{1}^{\perp}(s, t) d s,
$$

where $C=2 \pi R$ is the circumferential length of the accelerator ring. Since $\operatorname{Re} Z_{1}^{\perp}(\omega)>0$ implies an energy loss, the force leads the displacement $I_{0} a$ by $\frac{\pi}{2}$, and hence the factor $j$ in the definition of $Z_{1}^{\perp}$ above. The Lorentz factor $\beta=v / c$ is a convention. Inversely, the wake functions can be written in terms of the impedances:

$$
W_{m}(y)=\frac{j \beta}{2 \pi} \int_{-\infty}^{\infty} Z_{m}^{\perp}(\omega) e^{-j \omega y / v} d \omega, \quad W_{m}^{\prime}(y)=\frac{1}{2 \pi} \int_{-\infty}^{\infty} Z_{m}^{\|}(\omega) e^{-j \omega y / v} d \omega
$$


where the path of integration in both cases is below all the singularities of the impedances so as to guarantee causality. In terms of the time variable $t=-y / v$, the impedances can be rewritten in terms of the wake function as

$$
Z_{m}^{\|}(\omega)=\int_{-\infty}^{\infty} e^{-j \omega t} W_{m}^{\prime}(t) d t, \quad Z_{m}^{\perp}(\omega)=\frac{-j}{\beta} \int_{-\infty}^{\infty} e^{-j \omega t} W_{m}(t) d t,
$$

where* $W_{m}(t)$ is obtained from $W_{m}(y)$ by the substitution $y=-v t$. The inverse are

$$
W_{m}(t)=\frac{j \beta}{2 \pi} \int_{-\infty}^{\infty} Z_{m}^{\perp}(\omega) e^{j \omega t} d \omega, \quad W_{m}^{\prime}(t)=\frac{1}{2 \pi} \int_{-\infty}^{\infty} Z_{m}^{\|}(\omega) e^{j \omega t} d \omega .
$$

Note that the longitudinal wake is mostly the monopole $(m=0)$ wake $W_{0}^{\prime}$ and the transverse wake is mostly the dipole $(m=1)$ wake $W_{1}$, if the beam pipe cross section is close to circular and the particle path is close to the pipe axis. Thus, we are interested mostly in $Z_{0}^{\|}$and $Z_{1}^{\perp}$ only. For convenience, they may be written simply as $Z_{\|}$and $Z_{\perp}$. They have the dimensions of Ohms and Ohms/length, respectively. The impedances have the following properties: $^{\dagger}$

1. $Z_{m}^{\|}(-\omega)=\left[Z_{m}^{\|}(\omega)\right]^{*}$ and $Z_{m}^{\perp}(-\omega)=-\left[Z_{m}^{\perp}(\omega)\right]^{*}$.

2. $Z_{m}^{\|}(\omega)$ and $Z_{m}^{\perp}(\omega)$ are analytic with poles only in the upper half $\omega$-plane.

3. $Z_{m}^{\|}(\omega)=\frac{\omega}{c} Z_{m}^{\perp}(\omega)$,

for cylindrical geometry and each azimuthal harmonic including $m=0$.

4. $\operatorname{Re} Z_{m}^{\|}(\omega) \geq 0$ for all $\omega$ and $\operatorname{Re} Z_{m}^{\perp}(\omega) \gtreqless 0$ when $\omega \gtreqless 0$,

if the beam pipe has the same entrance cross section and exit cross section.

5. $\int_{0}^{\infty} d \omega \operatorname{Im} Z_{m}^{\perp}(\omega)=0$ and $\int_{0}^{\infty} d \omega \frac{\mathcal{I} m Z_{m}^{\|}(\omega)}{\omega}=0$ in most cases.

The first follows because the wake functions are real, the second from the causality of the wake functions, and the third from the Panofsky-Wenzel theorem between transverse and longitudinal electromagnetic forces. $\operatorname{Re} Z_{m}^{\|}(\omega) \geq 0$ is the result of the fact that the total energy of a particle or a bunch cannot be increased after passing through a section of the vacuum chamber where there are no accelerating external forces, while $\operatorname{Re} Z_{m}^{\perp}(\omega) \geq 0$ when $\omega>0$ follows from the Panofsky-Wenzel theorem. The fifth property follows from the assumption that $W_{m}(0)=0$.

Below are some examples of impedances:

*Obviously, $W_{m}^{\prime}(t) \equiv-v^{-1} d W_{m}(t) / d t$.

${ }^{\dagger}$ Although $Z_{0}^{\perp}$ may not have any physical meaning, it is a well-defined quantity mathematically. 


\section{Resonance}

A resonance can be represented, at least near the resonant frequency $\omega_{r}$, by a parallel $R L C$ circuit, giving the usual impedance

$$
Z(\omega)=\frac{R}{1+j Q\left(\frac{\omega}{\omega_{r}}-\frac{\omega_{r}}{\omega}\right)},
$$

where $\omega_{r}=(L C)^{-1 / 2}$ is the resonant frequency, and $Q=R \sqrt{L / C}$ is the quality factor. We can generalize this to the parallel impedance of the $m$ th harmonic:

$$
Z_{m}^{\|}(\omega)=\frac{R_{m, s h}}{1+j Q\left(\frac{\omega}{\omega_{r}}-\frac{\omega_{r}}{\omega}\right)},
$$

where the resonant frequency is $\omega_{r}=\left(L_{m} C_{m}\right)^{-1 / 2}$ and quality factor is $Q=R_{m, s h} \sqrt{C_{m} / L_{m}}$. Here, for the $m$ th multipole, the shunt impedance $R_{m, s h}$ is in $\mathrm{Ohms} / \mathrm{m}^{2 m}$, the inductance in henry $/ \mathrm{m}^{2 m}$, and the capacitance in farad $-\mathrm{m}^{2 m}$. The transverse impedance can now be obtained from the Panofsky-Wenzel theorem:

$$
Z_{m}^{\perp}(\omega)=\frac{c}{\omega} \frac{R_{m, s h}}{1+j Q\left(\frac{\omega}{\omega_{r}}-\frac{\omega_{r}}{\omega}\right)} .
$$

We observe that

(1) For small $Q \lesssim 1$, the impedances represent very broad resonances and will be used when the impedances are broadband. On the other hand, for large $Q$, they represent very sharp resonances.

(2) These impedances have two poles in the upper $\omega$-plane. When $Q$ is large, the impedances possess two narrow peaks near $\omega=\omega_{r}\left[ \pm \sqrt{\left|1-1 /\left(4 Q^{2}\right)\right|}+j /(2 Q)\right]$. The properties $Z_{m}^{\|}(-\omega)=\left[Z_{m}^{\|}(\omega)\right]^{*}$ and $Z_{m}^{\perp}(-\omega)=-\left[Z_{m}^{\perp}(\omega)\right]^{*}$ are evident.

\section{Resistive wall}

When the walls of the metallic beam pipe are not perfectly conducting, there will be a finite electric field $E$ at the walls, which decreases exponentially inside the walls of the pipe. Neglecting displacement current, which is important only at very high frequencies ${ }^{\ddagger}$ two of

\footnotetext{
${ }^{\ddagger}$ Displacement current can be neglected at frequencies $\omega \ll \sigma_{c} / \epsilon_{0} \approx \sigma_{c} Z_{0} c \approx 1.5 \times 10^{17} \mathrm{~Hz}$, where the conductivity of stainless steel $\sigma_{c}=1.35 \times 10^{6}(\Omega \text {-m })^{-1}$ is assumed.
} 
Maxwell equations are

$$
\vec{\nabla} \times \vec{E}=-\mu \frac{\partial \vec{H}}{\partial t}, \quad \vec{\nabla} \times \vec{H}=\vec{J}=\sigma_{c} \vec{E}
$$

where $\sigma_{c}$ is the conductivity and $\mu$ the permeability. Combining the two, we obtain

$$
\nabla^{2} \vec{E}=\mu \sigma_{c} \frac{\partial \vec{E}}{\partial t}, \quad \nabla^{2} \vec{H}=\mu \sigma_{c} \frac{\partial \vec{H}}{\partial t}
$$

Let $x>0$ be the distance into the metallic walls and $y$ be the direction along the wall. With the time dependency $e^{j \omega t}$,

$$
\frac{d^{2} E_{y}}{d x^{2}}=j \omega \mu \sigma_{c} E_{y}, \quad \frac{d^{2} H_{z}}{d x^{2}}=j \omega \mu \sigma_{c} H_{z}
$$

The solution is

$$
E_{y}=E_{y 0} e^{-[1+j \operatorname{sgn}(\omega)] x / \delta_{\text {skin }}}, \quad H_{z}=H_{z 0} e^{-[1+j \operatorname{sgn}(\omega)] x / \delta_{\text {skin }}}, \quad E_{y}=\frac{1+j \operatorname{sgn}(\omega)}{\sigma_{c} \delta_{\text {skin }}} H_{z},
$$

where

$$
\delta_{\text {skin }}=\sqrt{\frac{2}{|\omega| \mu \sigma_{c}}}
$$

is the skin depth at frequency $\omega$ or the penetration of field into the walls of the beam pipe, $E_{y 0}$ and $H_{z 0}$ are the electric and magnetic field at the surface of the pipe wall. The resistive loss portion of the impedance for a beam pipe of radius $b$ is

$$
\operatorname{Re} Z_{0}^{\|} \approx \frac{2 \pi R}{2 \pi b \delta_{\text {skin }} \sigma_{c}}
$$

and this is exactly the correct result if we follow a more accurate solution of the Maxwell equation by integrating into the walls of the beam pipe. Including the imaginary part, the total resistive wall impedance is, according to Eq. (2.19),

$$
Z_{0}^{\|}=[1+j \operatorname{sgn}(\omega)] \frac{R}{b \delta_{\text {skin }} \sigma_{c}} .
$$

The higher harmonic longitudinal impedance can be obtained in the same way by offsetting the beam and solving for the electric field at the wall. The distribution of longitudinal electric field on the surface of the beam pipe now depends on the azimuthal angle. Knowing the dipole longitudinal impedance, the transverse impedance is then obtained from the Panofsky-Wenzel theorem:

$$
Z_{1}^{\perp}=[1+j \operatorname{sgn}(\omega)] \frac{2 c R}{\omega b^{3} \delta_{\text {skin }} \sigma_{c}} .
$$




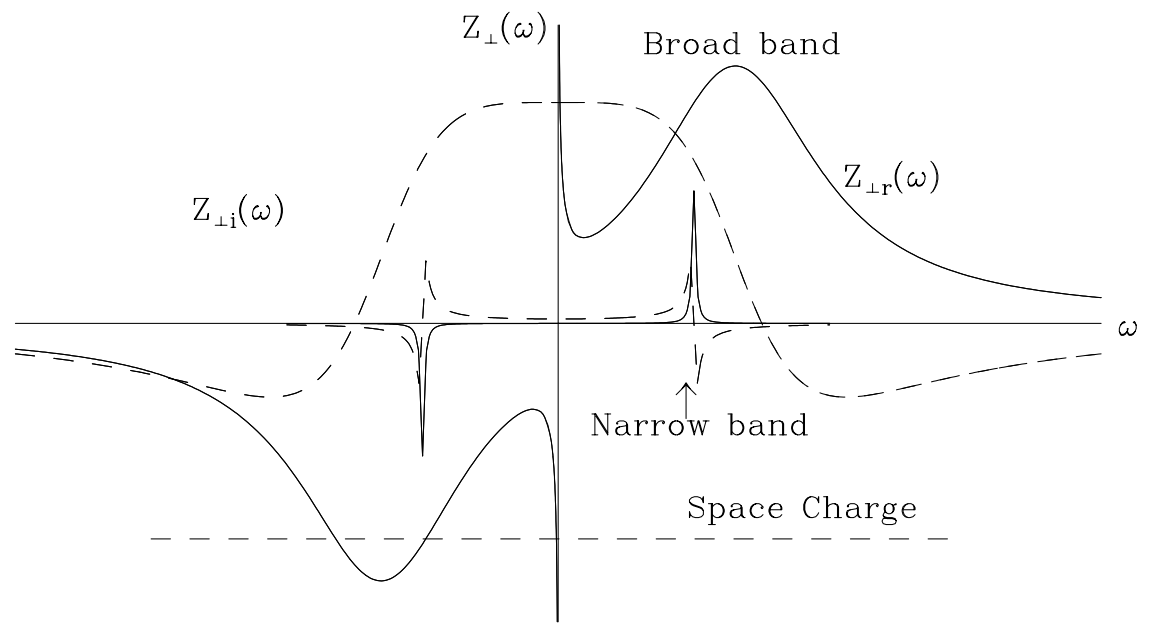

Figure 3: Schematic drawing of the transverse broadband and narrowband impedances. The real parts are shown in solid lines and the imaginary parts as dashed lines. A broadband and a narrowband impedance are represented by peaks in the real parts. The resistive wall impedance which peaks in the low-frequency region is included in the $\mathcal{R} e Z_{1}^{\perp}$ for the broadband. The space charge impedance is frequency independent. The schematic drawing of $\operatorname{Re} Z_{0}^{\|} / n$ and $\mathcal{I} m Z_{0}^{\|} / n$ should look similar.

Notice that

$$
Z_{1}^{\perp}=\frac{2 c}{b^{2}} \frac{Z_{0}^{\|}}{\omega},
$$

which looks like, but in fact is not the Panofsky-Wenzel relation. The two impedances belong to different azimuthal harmonics and should not have any relationship. Nevertheless, this relation has been used extensively as a convenient estimate of the transverse impedance from the longitudinal impedance, and sometimes good estimates are obtained. However, the space-charge impedances that we are going to discuss next violate this relation. The resistivewall impedance usually will not be strong enough to inflict severe instability of a single bunch. However, the transverse resistive-wall impedance reaches a very large value at low frequencies. ${ }^{\S}$ The betatron line at the smallest negative frequency acts like a sharp resonance and couples the transverse motion of all the bunches in the ring. This multi-bunch instability occurs in nearly every ring and is damped via a transverse mode damper and/or octupoles to supply a spread in betatron tunes. A schematic drawing of the transverse impedance of a ring is shown in Fig. 3, including narrowband resonance, resistive wall impedance, and space-charge resonance.

\footnotetext{
${ }^{\S}$ Eq. (2.23) shows that $Z_{1}^{\perp} \rightarrow \pm \infty$ as $\omega \rightarrow \pm 0$. More rigorous consideration shows that $Z_{1}^{\perp}$ eventually drops to zero when $\omega=0$.
} 


\section{Space-charge impedance}

Consider a particle beam with linear density $\lambda(s, t)$ traveling in the positive $s$ direction with velocity $v$ inside a cylindrical beam pipe of radius $b$ with infinitely-conducting walls. The beam is assumed to be rigid; therefore $\lambda(s, t)=\lambda(s-v t)$. We also assume that the beam is uniformly distributed transversely within a radius $a$ which varies very slowly longitudinally. We are interested in the electric field $E_{s}$ seen by the beam particles at the axis of the beam pipe. The radial electric field $E_{r}$ and azimuthal magnetic flux density $B_{\phi}$ at a radial distance $r$ are given by

$$
E_{r}=\left\{\begin{array}{l}
\frac{e \lambda r}{2 \pi \epsilon_{0} a^{2}} \\
\frac{e \lambda}{2 \pi \epsilon_{0} r}
\end{array} \quad B_{\phi}= \begin{cases}\frac{\mu_{0} e \lambda v r}{2 \pi a^{2}} & r \leq a, \\
\frac{\mu_{0} e \lambda v}{2 \pi r} & r \geq a,\end{cases}\right.
$$

where $e \lambda v$ is the beam current. Let us invoke Faraday's law

$$
\vec{\nabla} \times \vec{E}=-\frac{\partial}{\partial t} \vec{B}
$$

or in the integral form,

$$
\oint \vec{E} \cdot d \vec{\ell}=-\frac{\partial}{\partial t} \oint \vec{B} \cdot d \vec{A}
$$

In above, the closed path of integration of the electric field $\vec{E}$ is along two radii of the beam pipe at $s$ and $s+d s$ together with two length elements at the beam axis and the wall of the beam pipe, as illustrated in Fig. 4. The area of integration of the magnetic flux density $\vec{B}$

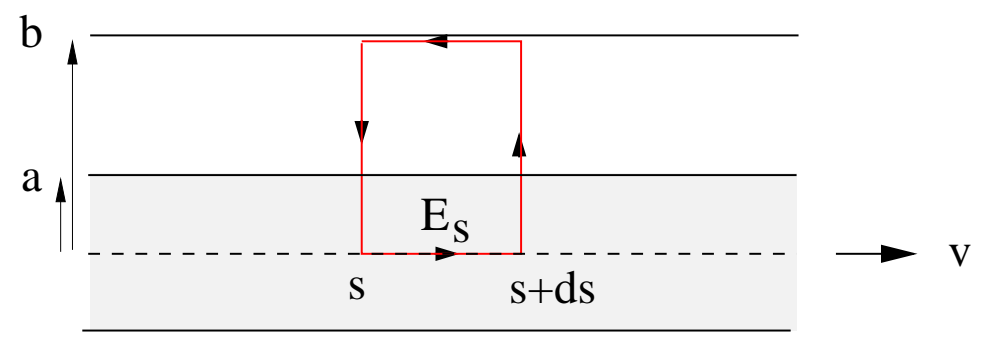

Figure 4: (color) Derivation of the space-charge longitudinal electric field $E_{s}$ experienced by a beam particle in a beam of radius $a$ in an infinitely conducting beam pipe of radius $b$. 
is the area enclosed by the closed path. Now, the left side of Eq. (2.27) becomes

$$
\text { L.S. }=E_{s} d s+\frac{e \lambda(s+d s-v t)}{2 \pi \epsilon_{0}}\left[\int_{0}^{a} \frac{r d r}{a^{2}}+\int_{a}^{b} \frac{d r}{r}\right]-E_{\mathrm{w}} d s-\frac{e \lambda(s-v t)}{2 \pi \epsilon_{0}}\left[\int_{0}^{a} \frac{r d r}{a^{2}}+\int_{a}^{b} \frac{d r}{r}\right],
$$

where $E_{\mathrm{w}}$ is the electric field at the wall of the beam pipe, while the right side

$$
\text { R.S. }=-\frac{\partial}{\partial t}\left(\frac{\mu_{0} e \lambda(s-v t) v}{2 \pi}\right)\left[\int_{0}^{a} \frac{r d r}{a^{2}}+\int_{a}^{b} \frac{d r}{r}\right] d s .
$$

Assumption has been made that the opening angle $1 / \gamma$ of the radial electric field is small compared with the distance $\ell$ over which the linear density changes appreciably, or $b / \gamma \ll \ell$. Here, $\gamma=E /\left(m c^{2}\right)$, where $E$ is the energy and $m$ is the rest mass of the beam particle. In terms of the the squared-bracketed expressions in Eqs. (2.28) and (2.29), we can define

$$
g_{0}=2\left[\int_{0}^{a} \frac{r d r}{a^{2}}+\int_{a}^{b} \frac{d r}{r}\right]=1+2 \ln \frac{b}{a}
$$

which is a geometric factor depending on the geometry of the beam and the beam pipe, and it will deviate from Eq. (2.30) if we relax, for example, the restriction of the transverse uniformity of the particle distribution. Combining the above, we arrive at

$$
E_{s}+\frac{e g_{0}}{4 \pi \epsilon_{0}} \frac{\partial \lambda}{\partial s}=v^{2} \frac{e \mu_{0} g_{0}}{4 \pi} \frac{\partial \lambda}{\partial s}+E_{\mathrm{w}}
$$

or

$$
E_{s}=-\frac{e g_{0}}{4 \pi \epsilon_{0} \gamma^{2}} \frac{\partial \lambda}{\partial s}+E_{\mathrm{w}}
$$

of which the first term on the right is the space-charge force experienced by a particle in a beam. Here, we see the near cancellation of the electric and magnetic forces. In the reduction from Eq. (2.30) to Eq. (2.32), use has been made of the relation $\epsilon_{0} \mu_{0}=c^{-2}$. The discontinuities of vacuum chamber are mostly inductive at low frequencies with total inductance $L$, leading to an induced wall electric field

$$
E_{\mathrm{w}}=\frac{L}{2 \pi R} \frac{d I_{\mathrm{w}}}{d t}=\frac{e \beta^{2} c^{2} L}{2 \pi R} \frac{\partial \lambda}{\partial s}
$$

where $I_{\mathrm{w}}$ is the image current at the surface of the pipe wall. The total longitudinal electric field at the pipe axis is therefore

$$
E_{s}=-e\left[\frac{g_{0}}{4 \pi \epsilon_{0} \gamma^{2}}-\frac{\beta^{2} c^{2} L}{2 \pi R}\right] \frac{\partial \lambda}{\partial s} .
$$

The linear distribution $\lambda$ consists of a uniform part $\lambda_{0}$ which has no $s$ variation and a perturbed part $\lambda_{1}$. The latter can be considered as a longitudinal harmonic wave,

$$
\lambda_{1}(s, t) \propto e^{j\left(\Omega_{c} t-n s / R\right)},
$$


where the collective frequency of the wave $\Omega_{c} \approx n \omega_{0}$ and $\omega_{0}=\beta c / R$ is the revolution frequency. The corresponding current is $I_{1}=e v \lambda_{1}$, and

$$
\frac{\partial \lambda_{1}}{\partial s}=-\frac{j n \lambda_{1}}{R}=-\frac{j n I_{1}}{e v R}
$$

The total voltage drop in one revolution on the beam is $\Delta U=-2 \pi R E_{s}$, giving a longitudinal impedance per harmonic

$$
\frac{Z_{0}^{\|}}{n}=\frac{\Delta U}{n I_{1}}=-j\left[\frac{g_{0} Z_{0}}{2 \beta \gamma^{2}}-\omega_{0} L\right]
$$

where $Z_{0}=\sqrt{\mu_{0} / \epsilon_{0}}=1 /\left(\epsilon_{0} c\right)=376.73 \Omega$ is called the vacuum impedance.

The longitudinal impedance per harmonic is frequency independent and rolls off only at very high frequencies when the wavelength drops to $\sim a / \gamma$. As an example, this amounts to a few tens of $\mathrm{GHz}$ for the Fermilab Main Injector. This space-charge force lengthens the bunch below transition and shortens it above transition and is the main cause of instability just after crossing transition. Typical values of the space-charge impedance at transition energy are listed in Table I.

Table I: Typical space-charge impedance at $\gamma=\gamma_{T}$.

\begin{tabular}{|l|l|l|l|l|l|}
\hline & AGS & RHIC & Fermilab BST & Fermilab MI & KEK PS \\
\hline$\gamma_{T}$ & 8.7 & 22.5 & 5.4 & 20.4 & 6.8 \\
$\left|Z_{0}^{\|} / n\right|(\Omega)$ & 13 & 1.5 & 30 & 2.3 & 20 \\
\hline
\end{tabular}

To compute the transverse space-charge impedance, let us shift the beam in the $x$ direction by an amount $x_{0}$, giving

$$
\left\{\begin{array}{l}
x \rightarrow x^{\prime}=x-x_{0} \\
r \rightarrow r^{\prime}=\sqrt{x^{\prime 2}+y^{2}}
\end{array}\right.
$$

where $x^{\prime}$ and $r^{\prime}$ are measured from center of beam while $x$ and $r$ are measured from center of beam pipe as illustrated in the left plot of Fig. 5. The current density is transformed

$$
\text { from } \quad i_{0}(r)=\frac{I_{0}}{\pi a^{2}} \Theta(a-r) \quad \text { to } \quad i(r, \psi)=i_{0}\left(r^{\prime}\right)=i_{0}(r)+i_{1}(r, \psi),
$$

where the dipole current density is, according to the right plot of Fig. 5,

$$
i_{1}(r, \psi)=\left.x_{0} \frac{d i_{0}\left(r^{\prime}\right)}{d x_{0}}\right|_{x_{0}=0}=\frac{I_{0}}{\pi a^{2}} x_{0} \frac{x}{r} \delta(a-r)=\frac{I_{0}}{\pi a^{2}} x_{0} \cos \psi \delta(a-r) .
$$

\footnotetext{
IIt will be shown later that $\Omega_{c}$ differs from $n \omega_{0}$ by the synchrotron frequency $\omega_{s}$ which is small and the synchrotron tune shift as a result of impedance which is small also.
} 
Let us compute the magnetic flux density induced by this dipole ring current at the center of the beam. Consider the current element at $\psi$ flowing longitudinally into the area element $a d \psi d r$. Magnetic flux density at the center of the beam in $z$ direction is

$$
\Delta B_{z}=\frac{\mu_{0} i_{1}(a, \psi) a d \psi d r}{2 \pi a} \cos \psi
$$

Summing up the contribution at $\pm \psi$ and $\pm(\pi-\psi), \Delta B_{z}$ is just 4 times. Thus contribution from the dipole current at the edge of the beam becomes

$$
\Delta B_{z}=\int_{0}^{\pi / 2} \frac{2 \mu_{0} I_{0} x_{0}}{\pi^{2} a^{2}} \cos ^{2} \psi d \psi=\frac{\mu_{0} I_{0} x_{0}}{2 \pi a^{2}} .
$$

This ring of dipole current induces a dipole image current in the walls of the beam pipe of radius $b$. The magnetic flux density induced by the dipole image current at the pipe center will be opposite to that from the ring of dipole current in the beam and is represented by Eq. (2.41) with $a \rightarrow b$. Total magnetic flux density induced on the beam is

$$
\Delta B_{z}=\frac{\mu_{0} I_{0} x_{0}}{2 \pi}\left(\frac{1}{a^{2}}-\frac{1}{b^{2}}\right) .
$$

The transverse impedance per unit length from magnetic field only is

$$
\begin{aligned}
Z_{1, \mathrm{mag}}^{\perp} & =\frac{j}{\beta e I_{0} x_{0}}\left\langle F_{1}^{\perp}\right\rangle=\frac{j}{\beta e I_{0} x_{0}} e v \Delta B_{z} \\
& =j \frac{\mu_{0} c}{2 \pi}\left(\frac{1}{a^{2}}-\frac{1}{b^{2}}\right)=j \frac{Z_{0}}{2 \pi}\left(\frac{1}{a^{2}}-\frac{1}{b^{2}}\right) .
\end{aligned}
$$

The transverse impedance per unit length from electric field, $Z_{1 \text {,elect }}^{\perp}$, is related to that from magnetic field by

$$
Z_{1, \text { mag }}^{\perp}=-\frac{1}{\beta^{2}} Z_{1, \text { elect }}^{\perp}
$$
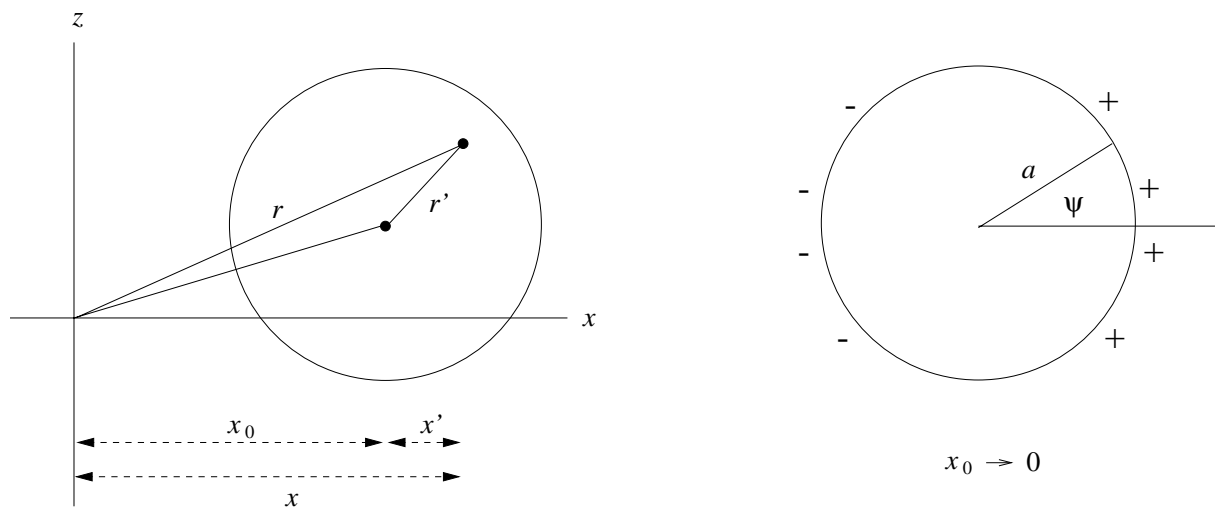

Figure 5: Left: Coordinates of a particle in a beam with the center offset by $x_{0}$ in the $x$ direction. Right: Cross section of dipole beam current when $x_{0} \rightarrow 0$. 
where one $\beta=v / c$ comes from the current and the second $\beta$ comes from the Lorentz force, just as what we got in the longitudinal case. Therefore, adding up both electric and magnetic contribution

$$
Z_{1}^{\perp}=-j \frac{Z_{0}}{2 \pi \beta^{2} \gamma^{2}}\left(\frac{1}{a^{2}}-\frac{1}{b^{2}}\right),
$$

where the first part is due to the space-charge self-force and the second part is due to images at the walls of the beam pipe. There are two comments:

(1) The monopole longitudinal and dipole transverse space-charge impedances obviously are not related by a Panofsky-Wenzel-like relation such as Eq. (2.24).

(2) The self-force part is related to the coherent self-force space-charge tune shift. It has been proven theoretically [2] and by simulation [3, 4] that beam instability is not caused by whether the spreads of the incoherent particle tunes overlap resonant lines in the tune plot, but by the coherent shift of the betatron tune into the resonant stop bands. Thus to ensure stability the coherent tune shifts, both horizontally and vertically, should be kept as small as possible.

\section{TRANSVERSE SPECTRUM WITH BETATRON OSCILLATION}

Consider a particle performing betatron oscillations with amplitude $A$ and tune $Q$. The transverse displacement is

$$
x(t)=A \cos \left(Q \omega_{0} t-\psi\right),
$$

where $\omega_{0}$ is the revolution frequency and $\psi$ is the betatron phase at $t=0$. Suppose that its azimuthal location along the accelerator ring is $\theta$ at $t=0$. The dipole moment of the particle picked up by a beam-position monitor at location $\theta_{\mathrm{BPM}}=0$ is the product of the transverse displacement and the longitudinal current,

$$
\begin{aligned}
d_{e}(t, \psi) & =e \omega_{0} A \cos \left(Q \omega_{0} t-\psi\right) \sum_{p=-\infty}^{\infty} \delta\left(\omega_{0} t-\theta-2 p \pi\right) \\
& =\frac{e \omega_{0} A}{2 \pi} \cos \left(Q \omega_{0} t-\psi\right) \sum_{n=-\infty}^{\infty} e^{j\left(n \omega_{0} t-n \theta\right)} \\
& =\frac{e \omega_{0} A}{4 \pi} \sum_{n=-\infty}^{\infty}\left\{e^{j\left[(n+Q) \omega_{0} t-n \theta-\psi\right]}+e^{j\left[(n-Q) \omega_{0} t-n \theta+\psi\right]}\right\} .
\end{aligned}
$$

The first term inside the curly brackets indicates upper sidebands around all the revolution harmonics, both positive and negative, while the second term indicates lower sidebands. This is illustrated in Fig. 6. As a result, the spectrum consists of betatron sidebands on both sides of the revolution harmonics. However, what we observe in a network analyzer or an oscilloscope are only positive frequencies, or the spectrum with the negative frequencies 

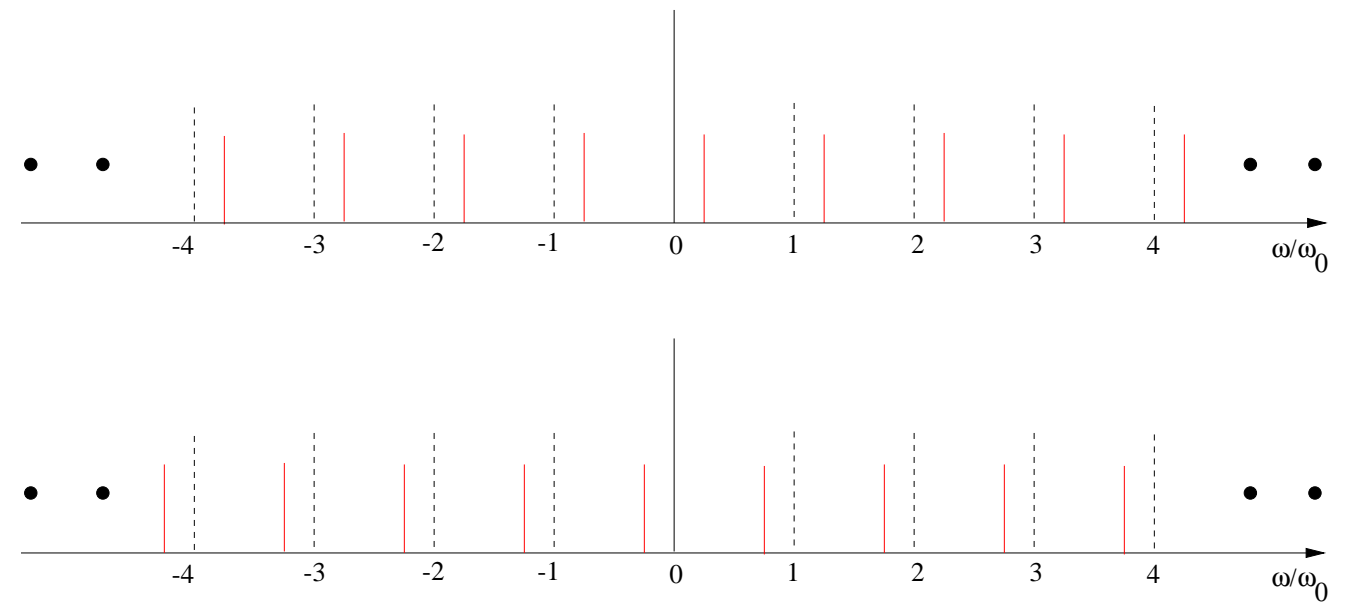

Figure 6: (color) Spectrum of a single particle executing betatron oscillations. Top: spectrum with upper betatron sidebands. Bottom: spectrum with lower betatron sidebands. Both spectra lead to exactly the same physical observation.

folded onto the positive frequencies about the zero frequency. It is clear that both the top and bottom spectra of Fig. 6 will lead to exactly the same physical observation. Because of this positive-negative-frequency symmetry, we need to study only the top spectrum with upper sidebands and discard the bottom spectrum with lower sidebands, or to study only the bottom spectrum and neglect the top one.

In fact, the second term in the curly brackets of Eq. (3.2) becomes $e^{-j\left[(n+Q) \omega_{0} t-n \theta-\psi\right]}$ if we make the substitution $n \rightarrow-n$. Then the dipole moment can be rewritten as

$$
d_{e}(t, \psi)=\frac{e \omega_{0} A}{2 \pi} \sum_{n=-\infty}^{\infty} \cos \left[(n+Q) \omega_{0} t-n \theta-\psi\right]
$$

Equivalently, this can also be written as

$$
d_{e}(t, \psi)=\frac{e \omega_{0} A}{2 \pi} \sum_{n=-\infty}^{\infty} \cos \left[(n-Q) \omega_{0} t-n \theta+\psi\right]
$$

This tells us again that, when both positive and negative frequencies are considered, upper sidebands and lower sidebands give the same physical picture. In most publications, the spectrum with the upper sidebands is chosen and we follow this convention here.

The angular phase velocity $\omega_{\mathrm{ph}}$ of a wave is just the ratio of the coefficient of $t$ to the coefficient of the coordinate $\theta$. In our case, for Eq. (3.3),

$$
\omega_{\mathrm{ph}}=\left(1+\frac{Q}{n}\right) \omega_{0} \quad \text { if } n \neq 0 .
$$




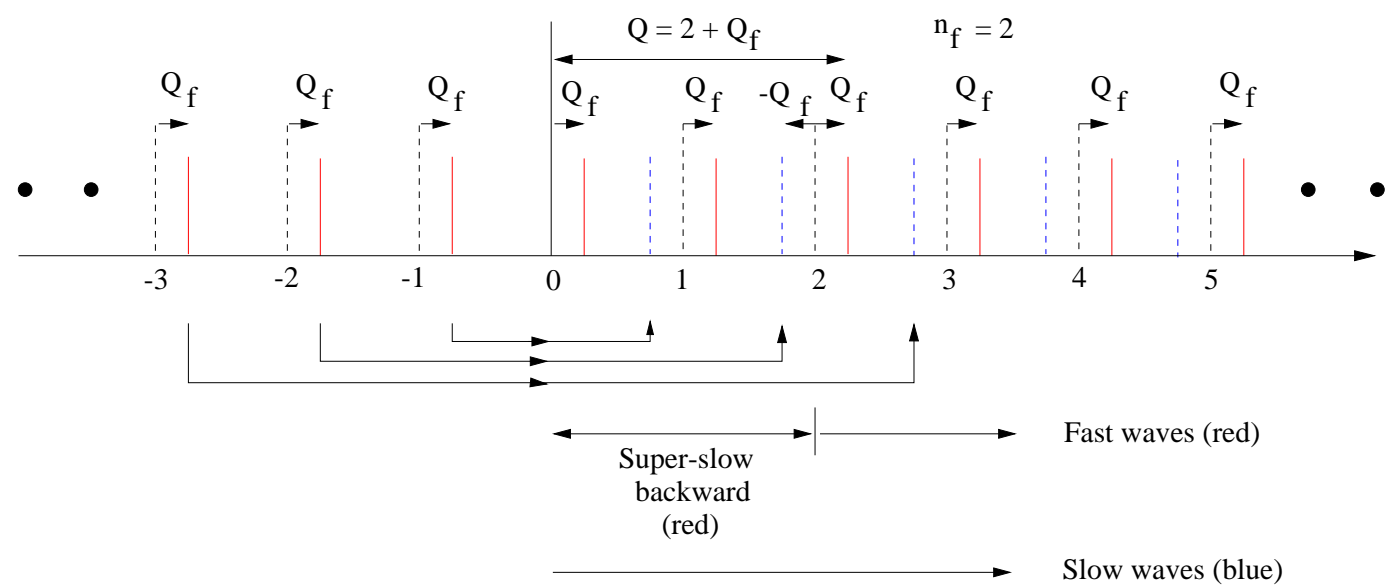

Figure 7: (color) Spectrum of a single particle executing betatron oscillations. Top: spectrum with upper betatron sidebands. Bottom: spectrum with lower betatron sidebands. Both spectra lead to exactly the same physical observation.

When $Q=0$, we see that the waves corresponding to all the revolution harmonics, positive and negative, have the same angular phase velocity $\omega_{0}$, which is equal to the angular velocity of the beam particle. With a nonzero betatron tune $Q$, we may think that the waves at sidebands corresponding to $n>0$ (upper sidebands seen in a network analyzer in an onlypositive-frequency language) will travel faster and those corresponding to $n<0$ will travel slower. The story is more complicated because the betatron tune has an integer part $n_{Q}$ and a noninteger part $Q_{f}$, or $Q=n_{Q}+Q_{f}$. An illustration is shown in Fig. 7 with $n_{Q}=2$ and $Q_{f}=0.25$. The illustration shows all the upper sidebands in red and the lower sidebands, reflected from the upper sidebands that have negative frequencies, in blue. Because $n_{Q}=2$, the upper sideband next to $n=0$ is actually associated with $n=-2$, while the one next to $n=1$ is associated with $n=-1$. Thus, these two upper sidebands correspond to waves with velocities $\omega_{\mathrm{ph}}=-0.125 \omega_{0}$ and $-1.25 \omega_{0}$ according to Eq. (3.5). These waves are so slow that they go backward. The next upper sideband next to $n=2$ is associated with $n=0$; the wave is stationary and not moving at all [Eq. (3.5) does not apply]. The upper sidebands next to $n=3,4, \cdots$ are associated with positive $n$ and they represent waves with velocities faster than $\omega_{0}$. All the lower sidebands (in blue) are associated with harmonics $n$ that are negative. Thus, they represent waves with velocities slower than $\omega_{0}$. Summing up

$$
\frac{\omega_{\mathrm{ph}}}{\omega_{0}}=1+\frac{Q}{n} \quad \text { with } \begin{cases}n>0 & \text { fast wave, } \\ n<0,|n|<Q & \text { backward wave } \\ n<0,|n|>Q & \text { slow wave }\end{cases}
$$

and $\omega_{\mathrm{ph}}=0$ or the wave is stationary when $n=0$. In the longitudinal spectrum we are going to study later, synchrotron sidebands correspond to waves with velocities that differ only slightly from the particle velocity, because the synchrotron tune is very much less than unity. On the other hand, we have seen that the velocities of waves corresponding to the betatron 
sidebands can differ very much from the particle velocity. It is important to distinguish slow waves from fast waves and backward waves, because, as we shall see later, only slow waves can be susceptible to instabilities. This is because $\mathcal{R} e Z_{1}^{\perp}(\omega) \gtrless 0$ when $\omega \gtrless 0$.

So far we have studied the betatron spectrum of a single particle. For many particles inside a beam, the transverse dipole moment is obtained by adding up the dipole moment $d_{e}(t, \psi)$ of all particles. Since the betatron phase $\psi$ is random among the particles, the dipole moment averages to zero, meaning that all these upper and lower betatron sidebands will not be visible to an oscilloscope.* They are only visible when excited by a transverse driving force, like a kicker, the transverse coupling impedance, etc. If a slow wave is excited and if there is no or not enough damping mechanism, the beam becomes unstable. For this reason, the lower betatron sidebands appear much more intense than the upper betatron sidebands.

\section{LONGITUDINAL SPECTRUM WITH SYNCHROTRON OSCILLATIONS}

Consider a particle with charge $e$ executing synchrotron oscillations of amplitude $\hat{\tau}$, frequency $\omega_{s}$, and phase $\psi$. The longitudinal current picked up by a wall-gap monitor at azimuthal location $\theta_{\text {wall }}=0$ can be represented by

$$
\begin{aligned}
I_{e}(t) & =e \sum_{\ell=-\infty}^{\infty} \delta\left[t-\hat{\tau} \cos \left(\omega_{s} t-\psi\right)-\ell T_{0}\right] \\
& =\frac{e \omega_{0}}{2 \pi} \sum_{n=-\infty}^{\infty} \exp \left\{j n\left[\omega_{0} t-\omega_{0} \hat{\tau} \cos \left(\omega_{s} t-\psi\right)\right]\right\},
\end{aligned}
$$

where $\omega_{0}=2 \pi / T_{0}$ is the revolution angular frequency. For convenience, we have omitted the azimuthal phase $\theta$ designating the location of the particle along the accelerator ring at time $t=0$. With the aid of

$$
e^{-j x \cos \psi}=\sum_{m=-\infty}^{\infty} j^{-m} J_{m}(x) e^{j m \psi}
$$

we can expand the current signal into azimuthal harmonics:

$$
I_{e}(t)=\frac{e \omega_{0}}{2 \pi} \sum_{n=-\infty}^{\infty} \sum_{m=-\infty}^{\infty} j^{-m} J_{m}\left(n \omega_{0} \hat{\tau}\right) e^{j\left(n \omega_{0}+m \omega_{s}\right) t} e^{j m \psi} .
$$

Here, we see discrete spectral lines at $\omega=n \omega+m \omega_{s}$ for $n=-\infty$ to $\infty$ and $m=-\infty$ to $\infty$, implying that there will be sidebands on both sides of all the revolution harmonics, either positive or negative. Since an oscilloscope records only positive frequencies, the lower

*They are visible in a Schottky scan. 
sidebands will be indistinguishable from the upper sidebands. Thus there will not be any loss of generality if we study only the upper sidebands and neglect the lower sidebands. This consideration is exactly the same as in the spectrum of a particle executing betatron oscillation but without synchrotron oscillation.

The Bessel functions in the summation determine the amplitude of the sidebands.* The synchrotron amplitude $\hat{\tau}$ is usually very much smaller than the revolution period. Let us take the Fermilab Tevatron as an example, the revolution period is $20.9 \mu$ s and a particle at the edge of a bunch of rms length $50 \mathrm{~cm}$ can have a synchrotron amplitude of $\hat{\tau} \approx 4 \mathrm{~ns}$, giving $\omega_{0} \hat{\tau} \approx 0.0012$. In this case, the lowest sidebands $m=1$ dominate at low frequencies. The revolution harmonics $(m=0)$ having amplitudes bounded by $J_{0}$ start dropping appreciably near harmonic $n \sim 800$, while the amplitudes of the $m=1$ sidebands increase first linearly with frequency under the envelope of $J_{1}$. At higher frequencies, the higher order sidebands $(m>1)$ will be observed. The $m=2$ sidebands can have larger amplitudes than the revolution harmonics $(m=0)$ and the $m=1$ sidebands when $J_{2}$ assumes a maximum near $n \sim 2500$. This is illustrated in Fig. 8, where $\omega_{0} \hat{\tau}=0.4$ has been used.

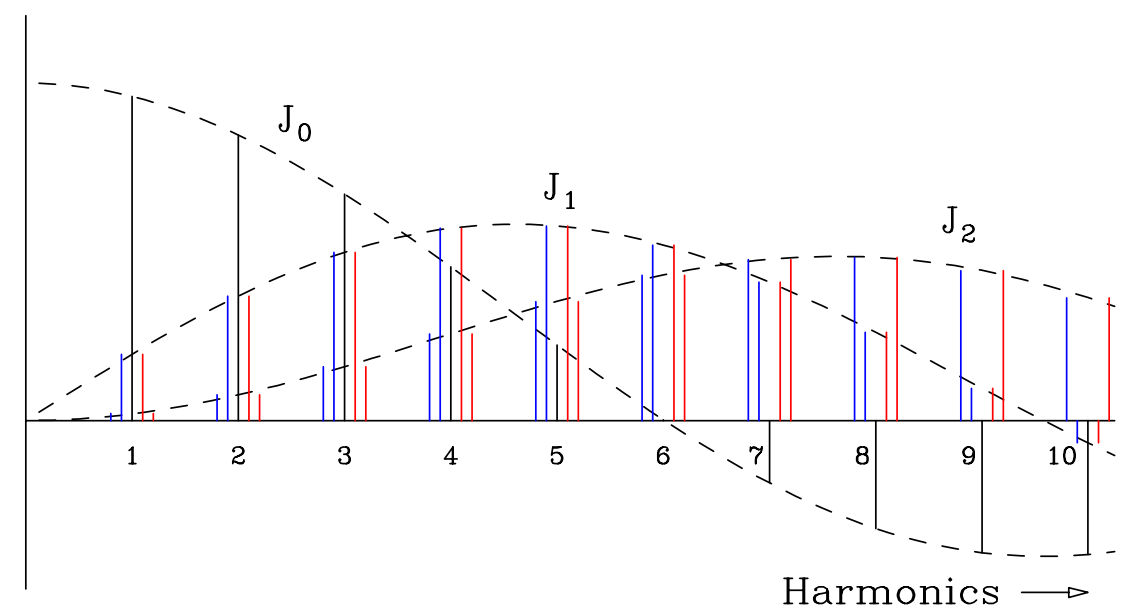

Figure 8: (color) Spectrum of a beam particle with synchrotron motion as observed by an oscilloscope with $\omega_{0} \hat{\tau}=0.4$. The upper sidebands associated with positive revolution harmonics are shown in red, while the upper sidebands associated with negative revolution harmonics are shown in blue and appear as lower sidebands when folded into positive frequencies. The revolution harmonics $(m=0)$ are bounded by Bessel function of order zero, the first synchrotron sidebands $(m=1)$ are bounded approximately by Bessel function of order one, and the second synchrotron sidebands $(m=2)$ are bounded approximately by Bessel function of order two. The phases of the sidebands have not been taken into account.

${ }^{*}$ Some authors approximate Eq. (4.1) by substituting $t=\ell T_{0}$ in the argument of the cosine. This will result in having $J_{m}\left(n \omega_{0} \hat{\tau}\right)$ in Eq. (4.3) changed to $J_{m}\left[\left(n \omega_{0}+m \omega_{s}\right) \hat{\tau}\right]$. In this approximation, the amplitude of the sidebands are bounded exactly by the Bessel functions. In the more exact expression of Eq. (4.3), however, these bounds are only approximate. 
It is important to point out that the Bessel functions have nothing to do with the linear distribution of the particle bunch because here we are dealing with only a point particle. The Bessel functions just reflect the synchrotron motion of the point particle. If we wish to study the signal from a bunch of particles, we need to multiply Eq. (4.3) by the distribution $\rho(\psi, \hat{\tau}, \theta)$ in the synchrotron phase $\psi$, the synchrotron oscillation amplitude $\hat{\tau}$, and particle azimuthal position $\theta$, and integrate over $\psi, \hat{\tau}$, and $\theta$. For example, if $\rho(\psi, \hat{\tau}, \theta)$ is random in $\psi$, the integral vanishes for all azimuthals except for $m=0$, or just the revolution harmonics. This is understandable because the bunch is smooth azimuthally. The revolution harmonics will still be visible because $\theta$ is localized for a bunch The distribution must be nonuniform in the synchrotron phase before some azimuthal sidebands can be excited. We also see that the sidebands will be broadened when signals are gathered from an ensemble of particles. This is due to a spread in energy among the particles and also the coupling impedance of the vacuum chamber. When excited below transition and an instability occurs, the lower synchrotron sidebands are unstable while the upper synchrotron sidebands are stable, and the other way around above transition. The reason is the same as in Robinson instability. [5]

\subsection{Coherent synchrotron modes}

The modes observed in a bunch of particles are called coherent modes. For a smooth bunch, the distribution function is $\rho(\hat{\tau}, \psi)=\rho_{0}(\hat{\tau})$. The current signal of this $N_{B}$-particle bunch picked up by a gap monitor is

$$
I_{0}(t)=\int I_{e}(t, \hat{\tau}, \psi) \rho_{0}(\hat{\tau}) \hat{\tau} d \hat{\tau} d \psi=I_{\mathrm{av}} \sum_{n=-\infty}^{\infty} A_{n, 0} e^{j n \omega_{0} t},
$$

where $I_{\mathrm{av}}=e N_{B} f_{0}$ is the average beam current, $f_{0}$ is the revolution frequency, and we assume that the integration over the azimuthal position $\theta$ has been performed. The amplitudes of the revolution harmonic signals $A_{n, 0}$ are the Hankel transforms of $\rho_{0}$,

$$
A_{n, 0}=2 \pi \int_{0}^{\infty} J_{0}\left(n \omega_{0} \hat{\tau}\right) \rho_{0}(\hat{\tau}) \hat{\tau} d \hat{\tau}
$$

If one measures the amplitudes of all the revolution harmonics, one can reconstruct the distribution $\rho(\hat{\tau})$ from the inverse Hankel transform.

With the $m$ th azimuthal mode excited, the bunch distribution becomes

$$
\rho(\hat{\tau}, \psi)=\rho_{0}(\hat{\tau})+\Delta \rho(\hat{\tau}, \psi)
$$

where

$$
\Delta \rho(\hat{\tau}, \psi)=\rho_{m}(\hat{\tau}) e^{j\left(\Omega_{c} t-m \psi\right)}
$$


and $\Omega_{c}$ is the coherent frequency. The signals of the bunch current become

$$
I(t)=I_{0}(t)+\Delta I(t)
$$

with the coherent current signal

$$
\Delta I(t)=\int I_{e}(t, \hat{\tau}, \psi) \Delta \rho(\hat{\tau} \psi) \hat{\tau} d \hat{\tau} d \psi=I_{\mathrm{av}} \sum_{n=-\infty}^{\infty} A_{n, m} e^{j\left(n \omega_{0}+m \omega_{s}+\Omega_{c}\right) t} .
$$

In above, the amplitude of the excited sideband, ${ }^{\dagger} A_{n, m}$, is the $m$ th order Hankel transform of $\rho_{m}$,

$$
A_{n, m}=2 \pi \int_{0}^{\infty} j^{-m} J_{m}\left(n \omega_{0} \hat{\tau}\right) \rho_{m}(\hat{\tau}) \hat{\tau} d \hat{\tau}
$$

Again, the collective perturbed distribution $\rho_{m}$ can be reconstructed through inverse Hankel transform when all $m$ th-azimuthal sideband amplitudes are measured by tuning a spectrum analyzer to each sideband. We can also obtain $A_{n, m}$ by taking the fast Fourier transform spectrum of the longitudinal beam profile digitized at fixed times during the onset of the coherent mode instability, for which a digital oscilloscope with a sizeable memory will be required.

To illustrate this, let us study the coherent synchrotron modes of a kicked beam. First assume a Gaussian equilibrium distribution and express it as a function of $\tau$ and $\dot{\tau} / \omega_{s}$,

$$
\rho_{0}(\hat{\tau})=\frac{1}{2 \pi \sigma_{\tau}^{2}} e^{-\hat{\tau}^{2} /\left(2 \sigma_{\tau}^{2}\right)}=\frac{1}{2 \pi \sigma_{\tau}^{2}} \exp \left[-\frac{\tau^{2}+\left(\dot{\tau} / \omega_{s}\right)^{2}}{2 \sigma_{\tau}^{2}}\right] .
$$

The beam is now kicked in phase by $\tau_{k}$. The initial distribution is obtained by substituting $\tau$ by $\tau+\tau_{k}$, or

$$
\rho\left(\tau, \frac{\dot{\tau}}{\omega_{s}}\right)=\frac{1}{2 \pi \sigma_{\tau}^{2}} \exp \left(-\frac{\tau_{k}^{2}}{2 \sigma_{\tau}^{2}}-\frac{\hat{\tau}^{2}}{2 \sigma_{\tau}^{2}}-\frac{\hat{\tau} \tau_{k}}{\sigma_{\tau}^{2}} \cos \psi\right),
$$

where $\hat{\tau}^{2}=\tau^{2}+\left(\dot{\tau} / \omega_{s}\right)^{2}$ and $\tau=\hat{\tau} \cos \psi$ have been used. Next invoke the identity

$$
e^{-x \cos \psi}=\sum_{m=-\infty}^{\infty}(-1)^{m} I_{m}(x) e^{j m \psi}
$$

to obtain

$$
\rho\left(\tau, \frac{\dot{\tau}}{\omega_{s}}\right)=\frac{1}{2 \pi \sigma_{\tau}^{2}} \exp \left(-\frac{\tau_{k}^{2}}{2 \sigma_{\tau}^{2}}-\frac{\hat{\tau}^{2}}{2 \sigma_{\tau}^{2}}\right) \sum_{m=-\infty}^{\infty}(-1)^{m} I_{m}\left(\frac{\hat{\tau}_{k}}{\sigma_{\tau}^{2}}\right) e^{j m \psi}
$$

where $I_{m}$ is the modified Bessel function of order $m$ having the property $I_{-m}=I_{m}$. We can now identify

$$
\rho_{m}(\hat{\tau})=\frac{1}{2 \pi \sigma_{\tau}^{2}} \exp \left(-\frac{\tau^{2}}{2 \sigma_{\tau}^{2}}-\frac{\hat{\tau}_{k}^{2}}{2 \sigma_{\tau}^{2}}\right)(-1)^{m} I_{m}\left(\frac{\hat{\tau}_{k}}{\sigma_{\tau}^{2}}\right)
$$

\footnotetext{
${ }^{\dagger}$ The phase $j^{-m}$ is unimportant, since it is the same for all $A_{n, m}$ 's.
} 
and the coherent mode integral in Eq. (4.10) becomes $^{\ddagger}$

$$
\begin{aligned}
A_{n, m} & =\frac{2 \pi j^{m}}{2 \sigma_{\tau}^{2}} e^{-\tau_{k}^{2} /\left(2 \sigma_{\tau}^{2}\right)} \int_{0}^{\infty} e^{-\hat{\tau}^{2} /\left(2 \sigma_{\tau}^{2}\right)} I_{m}\left(\frac{\hat{\tau} \tau_{k}}{\sigma_{\tau}^{2}}\right) J_{m}\left(n \omega_{0} \hat{\tau}\right) \hat{\tau} d \hat{\tau} \\
& =j^{m} e^{-\frac{1}{2}\left(n \omega_{0} \tau_{k}\right)^{2}\left(\frac{\sigma \tau}{\tau_{k}}\right)^{2}} J_{m}\left(n \omega_{0} \tau_{k}\right) .
\end{aligned}
$$

\subsection{Measurement of Coherent Synchrotron Modes}

An experimental measurement of coherent synchrotron modes was performed at the Cooler Ring of the Indiana University Cyclotron Facility (IUCF) a few years ago when holding the US Accelerator School. The ring had a revolution frequency of $f_{0}=1.03168 \mathrm{~Hz}$, a rf harmonic $h=1$, and a phase slip factor $\eta=-0.86$. The bunched beam contained about $5 \times 10^{8}$ protons at a kinetic energy of $45 \mathrm{MeV}$ and a rms length of about $20 \mathrm{~ns}$. The cycle time was $5 \mathrm{~s}$ while the injected beam was electron-cooled for about $3 \mathrm{~s}$. The beam was kicked longitudinally by phase-shifting the rf cavity wave form. The response time of the step phase shifts was limited primarily by the inertia of the rf cavities, which had a quality factor of about $Q=40$. The first kick was $\tau_{k}=90 \mathrm{ns,} \mathrm{or} \mathrm{equivalently} \omega_{0} \tau_{k}=0.58$. The synchrotron sideband power was observed from a spectrum analyzer tuned to the sideband. The sideband power of the first harmonic $f_{0}-f_{s}$, proportional to $\left|A_{1,1}\right|^{2}$, is shown in the upper trace of Fig. 9 and the sixth harmonic $6 f_{0}-f_{s}$, proportional to $\left|A_{6,1}\right|^{2}$, is shown in lower trace. The lower synchrotron sidebands were chosen because they are the more unstable ones below transition.

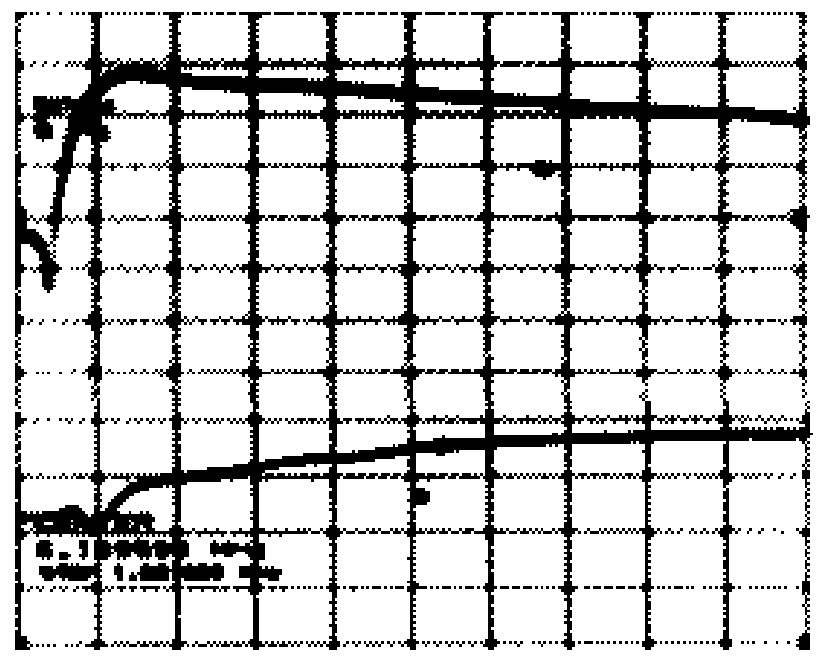

Figure 9: The synchrotron sideband power of the fundamental harmonic (top trace) and that of the 6th harmonic (lower trace), as measured by a spectrum analyzer tuned to the sidebands. The sidebands were excited by shifting the rf phase by $\tau_{k}=90 \mathrm{~ns}$. The amplitude of synchrotron oscillation was damped by electron cooling.

According to Eq. (4.16), the phase kick contributes

$$
A_{1,1} \sim e^{-0.0083} J_{1}(0.58) \text { and } A_{6,1} \sim e^{-0.299} J_{1}(3.48) .
$$

\footnotetext{
${ }^{\ddagger}$ Gradskteyn and Ryzhik 6.633.4: $\int_{0}^{\infty} e^{-\alpha x^{2}} I_{m}(\beta x) J_{m}(\gamma x) d x=\frac{1}{2 \alpha} e^{\left(\beta^{2}-\gamma^{2}\right) /(4 \alpha)} J_{m}\left(\frac{\beta \gamma}{2 \alpha}\right)$ if $\operatorname{Re} \alpha>0$
} and $\operatorname{Re} m>-1$. 
As a result, the sideband power of the fundamental harmonic is larger than that of the 6th harmonic by a factor of 6 , as is observed in the figure. As time goes on, the amplitude of synchrotron oscillation, initially at $\tau_{a}=\tau_{k}$, was damped by electron cooling. We see that the sideband power of the fundamental harmonic decreases and that of the 6th harmonic increases just as expected, because $J_{1}$ has its first maximum at 1.841 and its first zero at 3.832, as depicted in Fig. 10. The rf phase was then shifted to various values and the synchrotron side band power associated to each revolution harmonic was measured. Figure 11 shows the synchrotron sideband power as functions of frequency $\left(\omega \tau_{k}=n \omega_{0} \tau_{k}\right)$ for $\tau_{k}=53,90$, 100 , and $150 \mathrm{~ns}$. All data are normalized to the first peak $\left(n \omega_{0} \tau_{k} \approx 1.8\right.$ when $\tau_{k}=53$ and $n=1)$. Solid curves are $\left|A_{n, 1}\right|^{2}$ from Eq. (4.16) normalized to the peak. There are no other adjustable parameters. Satisfactory agreement of measurement with theory is observed.

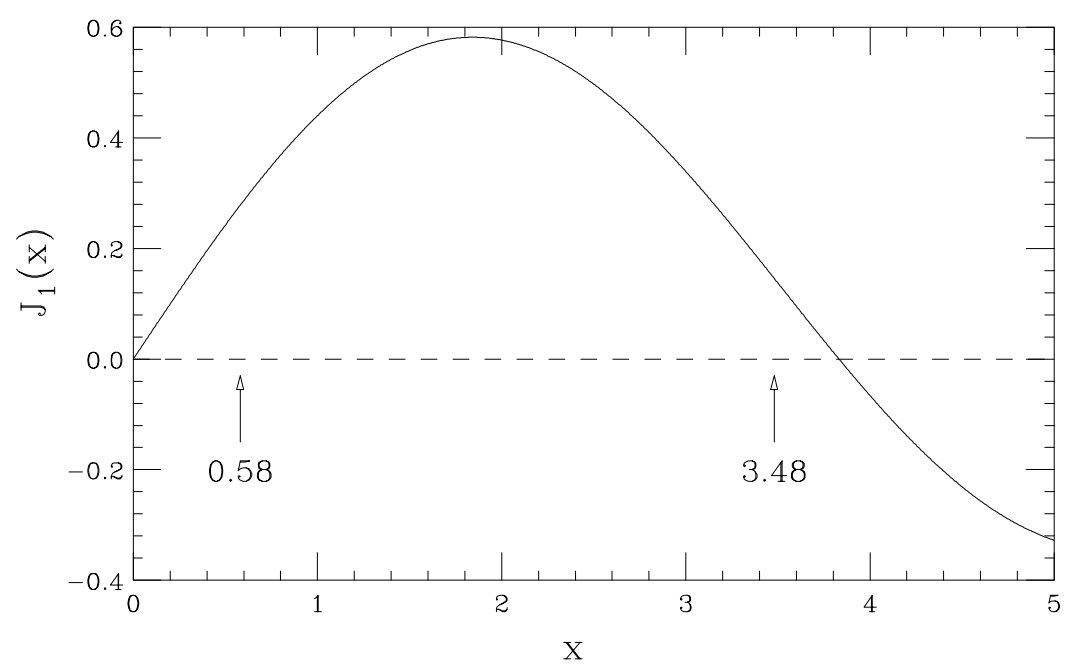

Figure 10: The bessel function of order one, $J_{1}(x)$, showing its first maximum at 1.841 and first zero at 3.832. Its argument is identified with $x=n \omega_{0} \tau_{a}$, where $\tau_{a}$ is the amplitude of the synchrotron oscillation at some later time after the rf phase shift. As $\tau_{a}$ decreases as a result of electron cooling, the power spectrum $\left|A_{1,1}\right|^{2}$ associated with $J_{1}\left(\omega_{0} \tau_{a}\right)$ decreases while the power spectrum $\left|A_{6,1}\right|^{2}$ associated with $J_{1}\left(6 \omega_{0} \tau_{a}\right)$ increases.

\section{TRANSVERSE INSTABILITY OF A COASTING BEAM}

Consider the $k$ th particle in a coasting beam executing betatron oscillation with frequency $Q_{k} \omega_{0}$. The transverse displacement $y_{k}$ satisfies the equation of motion

$$
\ddot{y}_{k}+\left(Q_{k} \omega_{0}\right)^{2} y_{k}=\frac{F_{\perp}}{\gamma m},
$$




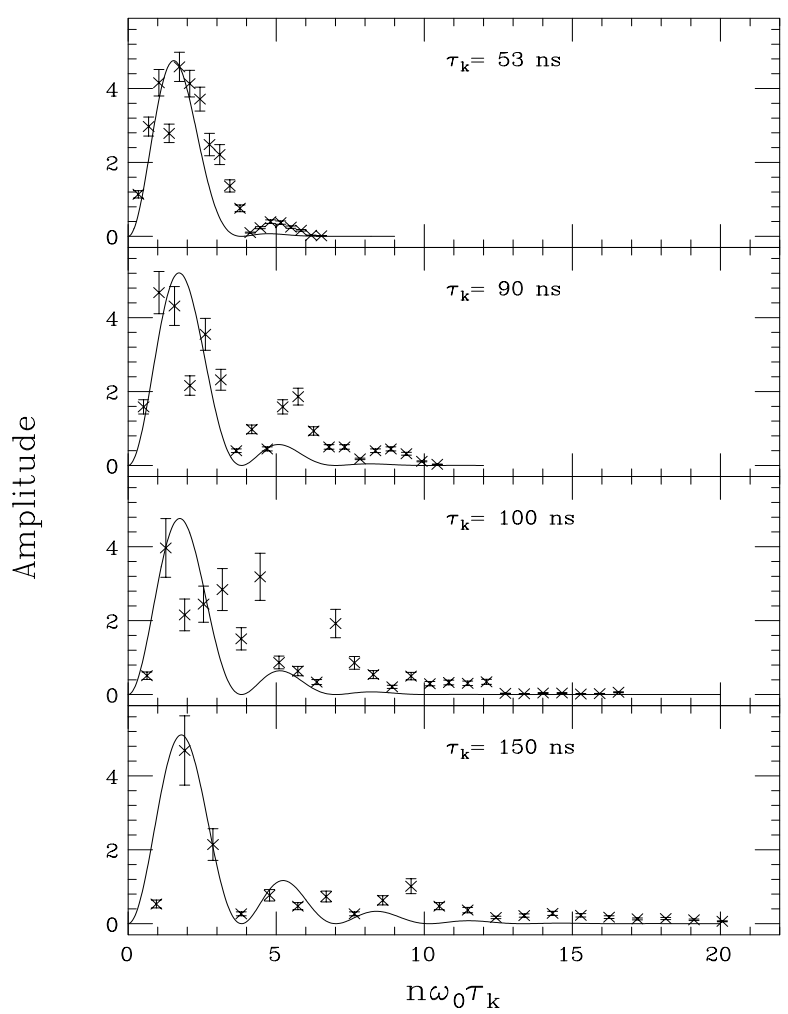

Figure 11: The initial synchrotron sideband power as functions of frequency $\omega \tau_{k}=n \omega_{0} \tau_{k}$ after $\mathrm{rf}$ phase shifts of $\tau_{k}=$ 53m 90, 100, and $150 \mathrm{~ns}$. The solid curves are the theoretical expectation normalized to the first peaks of the data. There are no other adjustable parameters.

where the double overdot corresponds to second derivative with respect to time, $m$ is the particle rest mass, and $\gamma$ is the Lorentz relativistic factor. Assuming that the transverse driving force $F_{\perp}$ is a result of a broadband impedance $Z_{1}^{\perp}$, we can write, according to Eq. (2.4),

$$
F_{\perp}=-\frac{e j I Z_{1}^{\perp}}{2 \pi R}\langle y\rangle
$$

where $\langle y\rangle$ is the transverse displacement of the beam center, $I\langle y\rangle$ is the dipole current, $e$ is the particle charge, and $R$ is the mean radius of the accelerator ring.

Let us assume the ansatz

$$
y_{k}=Y_{k} e^{j\left(\Omega_{c} t-n \theta\right)},
$$

where $n$ is a revolution harmonic, $\theta$ is the angle along the closed orbit of the accelerator, and $\Omega_{c}$ is the collective frequency to be determined later. This ansatz says nothing more than the fact that the revolution harmonic is a good eigennumber for the description of the collective motion, and its validity requires future verification. The time derivatives in Eq. (5.1) are total time derivatives, or

$$
\dot{y}_{k}=\frac{\partial y_{k}}{\partial t}+\dot{\theta} \frac{\partial y_{k}}{\partial \theta}=j\left(\Omega_{c}-n \omega_{0}\right) y_{k}
$$

Thus, Eq. (5.1) transforms to

$$
\left[\left(Q_{k} \omega_{0}\right)^{2}-\left(\Omega_{c}-n \omega_{0}\right)^{2}\right] y_{k}=-\frac{e j I Z_{1}^{\perp}}{2 \pi R}\langle y\rangle .
$$


The next step is to obtain an equation for the collective frequency independent of the transverse displacement $y_{k}$. The general way to accomplish this is to move the squared bracketed quantity on the left side of Eq. (5.5) to the denominator of the right side. We then multiply both sides by the transverse beam distribution $\rho(\xi)$. When we integrate over $\xi$, the left side becomes the mean transverse displacement of the beam, which cancels $\langle y\rangle$ on the right side. The result is a dispersion relation

$$
1=\frac{j \beta e I Z_{1}^{\perp}}{2 \pi R \gamma m} \int \frac{\rho(\xi)}{\left(\Omega_{c}-n \omega_{0}\right)^{2}-\left(Q \omega_{0}\right)^{2}} d \xi .
$$

where the betatron frequency $Q \omega_{0}$ is a function of $\xi$. This is called a dispersion relation because it relates the collective frequency of the beam to the impedance of the surrounding vacuum chamber. The success of obtaining the dispersion relation implies that the ansatz of Eq. (5.3) is indeed correct. The betatron frequency can be a function of momentum offset. It can also depend on the betatron amplitudes due to space-charge force, sextupoles and other magnetic multipoles of the accelerator lattice. All these dependencies are represented by the parameter $\xi$ and $\rho$ is just the beam transverse distribution in all these dependencies.

The denominator of the dispersion relation has two zeros

$$
\Omega_{c}-n \omega_{0}= \pm Q \omega_{0}
$$

The positive sign corresponds to looking at all the upper betatron sidebands of both the positive and negative revolution harmonics as illustrated in the upper plot of Fig. 6, while the lower sign corresponds to looking at all the lower sidebands in the lower plot of the figure. Since we know already that each of the two pictures will result in exactly the same physical observation, we can focus on one and discard the other. Since the two zeros are separated by twice the betatron tune which is a large number compared with the coherent tune shift to be determined, we can linearize the denominator of the dispersion relation near one of the zeroes. According to the convention of keeping only the upper sidebands, the linearization results in

$$
\left(\Omega_{c}-n \omega_{0}\right)^{2}-\left(Q \omega_{0}\right)^{2} \approx 2 Q \omega_{0}\left(\Omega_{c}-\omega_{n, w}\right) \quad \text { with } \quad \omega_{n, w}=(n+Q) \omega_{0} .
$$

The dispersion relation then takes the simpler form

$$
1=\frac{j \beta e I Z_{1}^{\perp}}{4 \pi R \gamma m Q \omega_{0}} \int \frac{\rho(\xi)}{\Omega_{c}-\omega_{n, w}(\xi)} d \xi .
$$

Now let us limit ourselves to the special case of betatron frequency spread versus momentum offset $\delta$ only. The betatron tune depends on $\delta$ through the chromaticity $C_{y}$ while the revolution frequency depends on $\delta$ through the phase slip parameter $\eta$ :

$$
\Delta Q=C_{y} \delta \quad \text { and } \quad \Delta \omega_{0}=-\eta \omega_{0} \delta
$$


We can therefore expand the betatron frequency in the form of

$$
\omega_{n, w}=\omega_{n, w_{0}}+\left[C_{y}-(n+Q) \eta\right] \omega_{0} \delta,
$$

where we have assigned the fractional off-momentum coordinate $\delta$ as the parameter $\xi$, and $\omega_{n, w_{0}}$ is the nominal value of $\omega_{n, w}$ for the on-momentum particle. As we shall see, the spread

in $\omega_{n, w}$ is the key of avoiding solutions which cause collective instabilities in the beam. Note that in Eq. (5.11) there is a revolution harmonic $n$ which minimizes the frequency spread of $\omega_{n, w}$ and even makes the spread vanish for some particular values of $C_{y}$.

\subsection{Zero Frequency Spread}

For a beam without momentum spread, the denominator of the dispersion relation in Eq. (5.9) is independent of the variable of integration and can be taken to the left side, giving the solution

$$
\Omega_{c}=\omega_{n, w_{0}}+j \frac{e \beta I Z_{1}^{\perp}}{4 \pi R \gamma m Q \omega_{0}} .
$$

With our time varying convention $e^{j \omega t}$, any negative imaginary part of the frequency will be an exponential growth. While $\operatorname{Im} Z_{1}^{\perp}$ contributes to a frequency shift, $\operatorname{Re} Z_{1}^{\perp}$ at negative frequency* contributes to a growth. We recall from our discussion of Fig. 7 that all betatron sidebands that are of negative values correspond to slow waves. We therefore reach the conclusion that all slow waves that support a real part of the transverse impedance are intrinsically unstable unless there exists some damping mechanism.

\subsection{Finite Frequency Spread}

With a finite frequency spread, the integral in the dispersion relation must be performed to decide whether there is an unstable solution. When the transverse impedance $Z_{1}^{\perp}$ is given, the dispersion relation is an eigenequation, with the collective frequency $\Omega_{c}$ the eigenvalue and the particle displacements $y(\xi)$ the eigenfunction. Let us define the parameters

$$
V+j U=\frac{e \beta I Z_{1}^{\perp}}{4 \pi R \gamma m Q \omega_{0}},
$$

which are just representation of the impedance:

$$
V \sim \operatorname{Re} Z_{1}^{\perp} \quad \text { and } \quad U \sim \operatorname{Im} Z_{1}^{\perp} .
$$

The dispersion relation is now written as

$$
(-U+j V)^{-1}=\int \frac{\rho(\xi)}{\Omega_{c}-\omega_{n, w}(\xi)} d \xi
$$

${ }^{*}$ Recall that $\operatorname{Re} Z_{1}^{\perp}$ is positive at positive frequencies and negative at negative frequencies. 
To analyze the stability of the beam, we first fix one value for the imaginary part of the collective frequency $\Omega_{c}$ (the growth rate) and vary the real part (the real frequency shift). For each $\mathcal{R} e \Omega_{c}$, the dispersion integral is solved and a pair of values of $U$ and $V$ are obtained. In this way, an equal-growth contour is drawn in $U-V$ plane. One such contour corresponding to $\mathcal{I} m \Omega_{c}=0-$ is of particular interest, because it demonstrates the edge of stability and we call it the stability contour. When these contours are drawn, we can read out readily whether the beam will be stable and if not the growth rate once the impedance of the vacuum chamber (corresponding to one point in the $U-V$ plane) is known.

To demonstrate this, let us consider a coasting beam with a Gaussian momentum-spread distribution

$$
\rho(\delta)=\frac{1}{\sqrt{2 \pi} \sigma_{\delta}} e^{-\delta^{2} /\left(2 \sigma_{\delta}^{2}\right)},
$$

where $\delta$ is the fractional momentum offset of some particle. With $\omega_{n, w}(\delta)$ given by Eq. (5.11), the dispersion relation can be solved in terms of the complex error function

$$
w(z)=e^{-z^{2}} \operatorname{erfc}(-j z)=\frac{j}{\pi} \int_{-\infty}^{\infty} \frac{e^{-t^{2}}}{z-t} d t,
$$

where erfc is the complementary error function. Thus the solution of the dispersion relation can be written as

$$
(v+j u)^{-1}=w\left(\frac{\Omega_{c}-\omega_{n, w_{0}}}{\sigma_{\omega}}\right),
$$

where

$$
\sigma_{\omega}=\sqrt{2}\left[C_{y}-(n+Q) \eta\right] \omega_{0} \delta
$$

is the rms betatron frequency spread of the beam for harmonic mode $n$ and

$$
u=\frac{\sqrt{\pi} U}{\sigma_{\omega}} \text { and } \quad v=\frac{\sqrt{\pi} V}{\sigma_{\omega}} .
$$

The contours for $\operatorname{Im} \Omega_{c}=0$ and $-0.5 \sigma_{\omega}$ are shown in Fig. 12. Since only the slow waves will be unstable, the first left contour represents growth rate of $0.5 \sigma_{\omega}\left(\mathcal{I} m \Omega_{c}=-0.5 \sigma_{\omega}\right)$ while the second left contour represents the threshold. Any region to the right of this threshold curve (including the two contours on the right) is stable. The rectangular symbols represent coherent frequency shift of $\Omega_{c}-n \omega_{0}= \pm \sigma_{\omega}$ (inner ones) and $\pm 2 \sigma_{\omega}$ (outer ones). Without any betatron frequency spread (or momentum spread) in the beam, we learned from the previous section that any small amount of $\mathcal{R} e Z_{1}^{\perp}$ at negative frequency (any negative $v$ ) will be able to drive the beam unstable. Now with a Gaussian betatron frequency spread, when the betatron frequency shift is $\pm \sigma_{\omega}$, a negative $v$ of $\sim 3$ units is required to drive the beam to instability. When the frequency shift becomes $\pm 2 \sigma_{\omega}$, a negative $v$ of $\sim 0.8$ is required to drive the beam to instability. This implies that there has been some damping which counteracts the impedance to stabilize the beam. This damping mechanism is called 


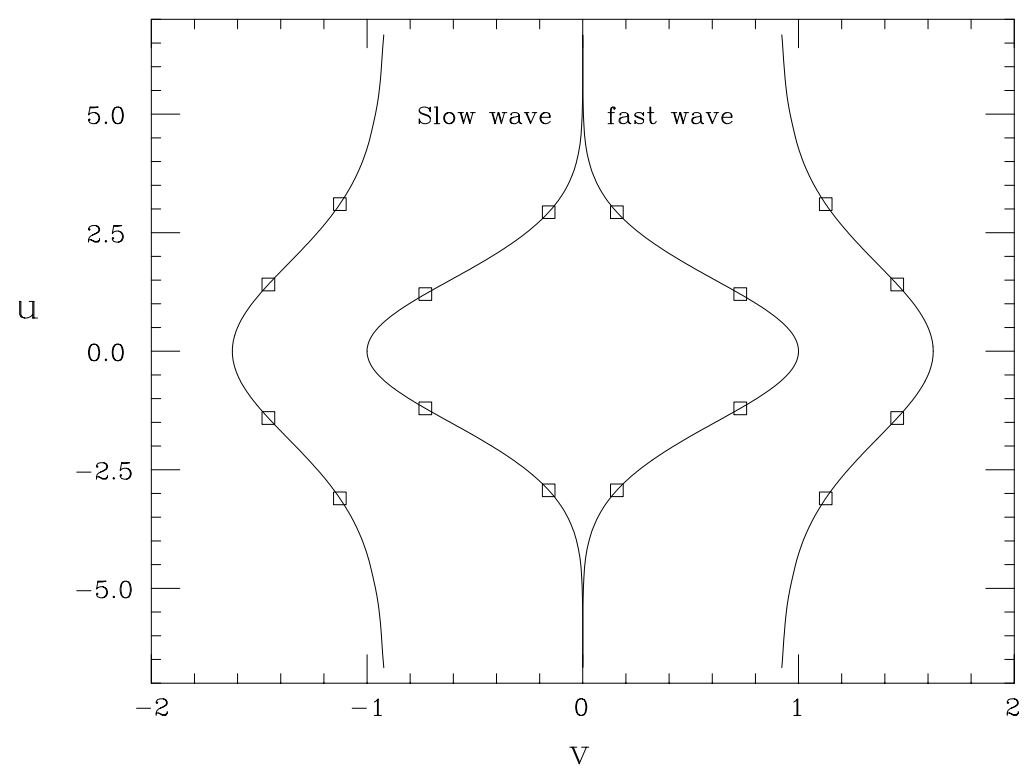

Figure 12: Normalized plots of contours corresponding to growth rates $\mathcal{I} m \Omega_{c}=-0.5 \sigma_{\omega}$ and $\mathcal{I} m \Omega_{c}=0$ (the first left and second left curves) for a Gaussian distribution of the momentum offset of a coasting beam. The rectangular symbols represent three coherent frequency shifts of $\operatorname{Re}\left(\Omega_{c}-n \omega_{0}\right)= \pm \sigma_{\omega}$ and $\pm 2 \sigma_{\omega}$.

Landau damping which we are going to study in the next section. In general whenever the betatron frequency shift is within the distribution $\rho$, there will be Landau damping to help fighting against the impedance driving force. Landau damping becomes smaller when the frequency shifts towards the edge of the distribution $\rho$, and finally vanishes when the shift is outside the distribution.

\subsection{A Model of Collective Motion}

The dispersion relation can also be solved by diagonalizing a matrix. Consider an ensemble of $N$ macroparticles. The $k$ th particle is in a certain distribution $\rho_{k}$ and has a transverse offset $Y_{k}$. Thus, the offset of the beam center is

$$
\langle Y\rangle=\sum_{\ell=1}^{N} \rho_{\ell} Y_{\ell} \quad \text { with } \quad \sum_{\ell=1}^{N} \rho_{\ell}=1
$$

The wake force drives the center of the beam. The equation of motion of the $k$ th particle can be rewritten as

$$
\left(\Omega_{c}-\omega_{n, w_{k}}\right) Y_{k}=W \sum_{\ell=1}^{N} \rho_{\ell} Y_{\ell},
$$

where $W$ is complex and can be represented by

$$
W=j A Z_{1}^{\perp}=-A \mathcal{I} m Z_{1}^{\|}+j A \mathcal{R e} Z_{1}^{\perp},
$$


with $A$ being a positive constant. There are $N$ collective modes and their respective collective mode frequencies $\omega$ can be obtained by solving the eigenvalue-eigenfunction matrix equation:

$$
\left(\begin{array}{cccc}
\Omega_{c}-\omega_{n, w_{1}} & 0 & \cdots & 0 \\
0 & \Omega_{c}-\omega_{n, w_{2}} & \cdots & 0 \\
\ldots & \cdots & \cdots & \cdots \\
0 & 0 & \cdots & \Omega_{c}-\omega_{n, w_{N}}
\end{array}\right)\left(\begin{array}{c}
Y_{1} \\
Y_{2} \\
\ldots \\
Y_{N}
\end{array}\right)=W\left(\begin{array}{cccc}
\rho_{1} & \rho_{2} & \cdots & \rho_{N} \\
\rho_{1} & \rho_{2} & \cdots & \rho_{N} \\
\cdots & \cdots & \cdots & \cdots \\
\rho_{1} & \rho_{2} & \cdots & \rho_{N}
\end{array}\right)\left(\begin{array}{c}
Y_{1} \\
Y_{2} \\
\ldots \\
Y_{N}
\end{array}\right) .
$$

Let us consider the following special cases:

(1) No spread in betatron frequency.

Without any spread, we can write

$$
\omega_{n . w k}=\omega_{n . w 0} .
$$

Equation (5.22) can be solved easily by multiplying on both sides by $\rho_{k}$ and summing over $k$. The solution is

$$
\Omega_{c}=\omega_{n, w_{0}}+W,
$$

which is unstable for the slow waves because $\operatorname{Im} W \propto \mathcal{R} e Z_{1}^{\perp}$, agreeing with what we studied before.

(2) Space-charge tune spread

We learn that a spread in the particle oscillation frequency provides Landau damping. However, we want to show here that space-charge tune spread will not provide Landau damping. For a cylindrically symmetric beam, the space-charge force on a particle at an offset $r$ is

$$
F_{\mathrm{sp} \mathrm{ch}} \propto \frac{1}{2 \pi r} \int_{0}^{r} \rho\left(r^{\prime}\right) 2 \pi r^{\prime} d r^{\prime}=f(r) r,
$$

where

$$
f(r)=\frac{1}{2 \pi r^{2}} \int_{0}^{r} \rho\left(r^{\prime}\right) 2 \pi r^{\prime} d r^{\prime} .
$$

For example, in the bi-Gaussian distribution $\rho(r)=e^{-r^{2} /\left(2 \sigma_{r}^{2}\right)} /\left(2 \pi \sigma_{r}^{2}\right)$,

$$
f(r)=\frac{1}{2 \pi}\left(1-e^{-r^{2} /\left(2 \sigma_{r}^{2}\right)}\right) \stackrel{r \rightarrow 0}{\longrightarrow} \frac{1}{4 \pi \sigma_{r}^{2}} .
$$

Including this incoherent force, the equation of motion becomes

$$
\left(\Omega_{c}-\omega_{n, w_{0}}\right) Y_{k}+f_{k} \Delta \Omega\left(Y_{k}-\langle Y\rangle\right)=W \sum_{\ell=1}^{N} \rho_{\ell} Y_{\ell}
$$


since the space-charge force is measured with respect to the center of the beam. In above, $\Delta \Omega$ is proportional to the space-charge tune spread. Collecting all the terms proportional to $\langle Y\rangle$ to the right side,

$$
\left(\Omega_{c}-\omega_{n, w_{0}}+f_{k} \Delta \Omega\right) Y_{k}=\left(W-f_{k} \Delta \Omega\right)\langle Y\rangle .
$$

We can eliminate $\langle Y\rangle$ to obtain the dispersion relation

$$
1=\sum_{\ell=1}^{N} \rho_{k} \frac{W+f_{k} \Delta \Omega}{\Omega_{c}-\omega_{n, w_{0}}+f_{k} \Delta \Omega} .
$$

It is clear the solution is

$$
\Omega_{c}-\omega_{n, w_{0}}=W .
$$

Thus, the space-charge tune spread does not affect the collective mode frequencies at all. This demonstration is very general; it just depends on the fact that the space-charge force is measured from the center of the beam and is independent of the distribution $f_{k}$, nor does it matter whether the distribution is discrete or continuous. On the other hand, the displacement $Y_{k}$ is measured from the closed orbit of the machine.

(3) Landau damping

Now consider the same equation but with the tune-spread force measured from the closed orbit,

$$
\left(\Omega_{c}-\omega_{n, w_{0}}\right) Y_{k}+f_{k} Y_{k}=W \sum_{\ell=1}^{N} \rho_{\ell} Y_{\ell} .
$$

Without the impedance-driving force on the right side, the betatron frequency of the individual particles are

$$
\Omega_{c}=\omega_{n, w_{0}}+f_{k} .
$$

Every particle is oscillating with a slightly different frequency. On the other hand, the impedance-driving force is introducing a growth rate proportional to - $\operatorname{Im} W$ for the coherent amplitude. If the frequency spread is larger than the growth rate, the phases of oscillation of the particles cannot be kept roughly the same for one growth time. In other words, the collective growth will not be able to materialize. The rule of thumb for Landau damping is: the spread in oscillation frequency must be larger than the growth rate without damping. The collective mode frequencies can be obtained by diagonalizing the matrix equation.

One must understand that the mechanism of Landau damping is the shifting of energy onto one collective mode from the damping of all other modes. Therefore, there must be many collective modes or many particles inside the beam so that energy can be transferred from one mode to another. For example, if there are only two particles with different unperturbed 
betatron frequencies, there will not be any Landau damping no matter how large the tune split is between the two particles. This can be demonstrated easily. The matrix equation now becomes

$$
\left(\begin{array}{cc}
\Delta \omega & 0 \\
0 & \Delta \omega+\Delta \Omega
\end{array}\right)\left(\begin{array}{c}
Y_{1} \\
Y_{2}
\end{array}\right)=W\left(\begin{array}{cc}
\frac{1}{2} & \frac{1}{2} \\
\frac{1}{2} & \frac{1}{2}
\end{array}\right)\left(\begin{array}{c}
Y_{1} \\
Y_{2}
\end{array}\right)
$$

where $\Delta \Omega$ is the frequency split, $\rho_{k}=\frac{1}{2}$ is the particle density, and $\Delta \omega=\Omega_{c}-\omega_{n, w_{0}}$. The collective frequencies are

$$
\Delta \omega=\frac{W-\Delta \Omega}{2} \pm \sqrt{\frac{W^{2}+\Delta \Omega^{2}}{4}} .
$$

If we include only the unstable part of $W$, i.e., writing $W=j \operatorname{Im} W$, the collective frequencies become

$$
\Delta \omega=\frac{j \operatorname{Im} W-\Delta \Omega}{2} \pm \sqrt{\frac{\Delta \Omega^{2}-(\operatorname{Im} W)^{2}}{4}} .
$$

We see that no matter how big the frequency split $\Delta \Omega$ is, there is always an unstable solution.

\section{LANDAU DAMPING}

Consider the ensemble of particles with intrinsic betatron frequency $\omega_{\beta}=Q \omega_{0}$ driven by an external force of frequency $\omega$ and strength $\hat{F}$. The equation of motion of a particular particle is

$$
\ddot{y}+\omega_{\beta}^{2} y=\hat{F} \sin \omega t .
$$

This solution of the equation is usually singular when the frequency of the driving force approaches the intrinsic frequency of the particles. However, when the initial conditions $y=y_{0}$ and $\dot{y}=\dot{y}_{0}$ are specified, the solution

$$
y(t)=\frac{\hat{F}}{\omega_{\beta}^{2}-\omega^{2}}\left(\sin \omega t-\frac{\omega}{\omega_{\beta}} \sin \omega_{\beta} t\right)+y_{0} \cos \omega_{\beta} t+\frac{\dot{y}_{0}}{\omega_{\beta}} \sin \omega_{\beta} t
$$

becomes well-behaved when $\omega=\omega_{\beta}$. The terms involving the initial conditions will diminish as a result of decoherence in the presence of a spread in the betatron frequency. They do not participate in the dynamic interaction of the beam particles and will be neglected at this moment.

What we are interested in is the situation when the driving frequency $\omega$ is very close to the betatron frequency $\omega_{\beta}$ of the particles, i.e., $\omega_{\beta}=\omega+\epsilon$ with $|\epsilon| \ll \omega$. The solution is

$$
y(t) \approx \frac{\hat{F}}{2 \omega}\left[\frac{1-\cos (\epsilon t)}{\epsilon} \sin \omega t-\frac{\sin \epsilon t}{\epsilon} \cos \omega t\right],
$$


where we have separated the fast-oscillating parts, namely $\omega t$, from the slowly oscillating parts, namely $\epsilon t$. The first term is in phase with the driving force and the second term $90^{\circ}$ out of phase. Thus energy is exchanged through the second term. The two slowly varying functions

$$
p(\epsilon)=\frac{1-\cos \epsilon t}{\epsilon} \quad \text { and } \quad d(\epsilon)=\frac{\sin \epsilon t}{\epsilon}
$$

are illustrated in Fig. 13. In particular, $d(\epsilon)$ controls how the energy is transferred. At the beginning (time $t$ is small), the main peak of $d(\epsilon)$ is wide enough to include the the betatron frequency $\omega_{\beta}=\omega+\epsilon$ of the particle, and energy is absorbed by the particle resulting in an increase in oscillation amplitude. As time increases to $t>\pi /|\epsilon|$, the main peak of $d(\epsilon)$ shrinks to the point that the particle betatron frequency falls outside the peak. The particle then no longer is able to absorb energy from the driving force. Instead, it gives out its oscillation energy back to the driving force. Therefore, as time goes on, more and more particles will not be driven by the force and only those few particles having betatron frequencies extremely close to the driving frequency, or extremely small $|\epsilon|$, will continue to receive energy from the driving force. In other words, collective motion of the whole particle beam is no longer possible. This mechanism of inability to increase the oscillation amplitude is called Landau damping. An illustration is shown in Fig. 14, where the driving frequency is set at $\omega=0.75 \mathrm{~Hz}$, while the driven oscillations of three particles having betatron frequencies
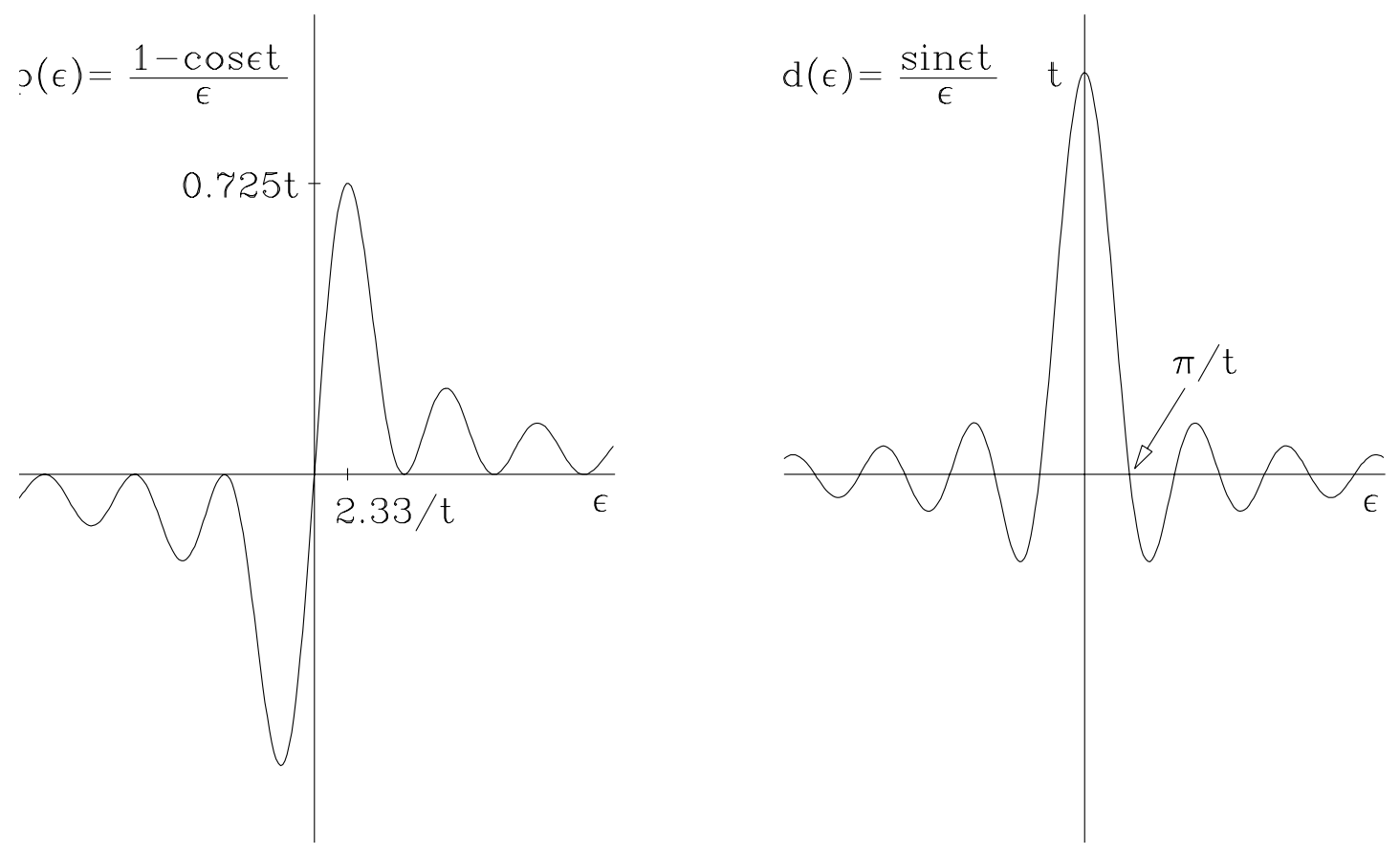

Figure 13: Plots of the two slowly varying functions, $p(\epsilon)$ and $d(\epsilon)$, with time $t$ being a parameter. As $t \rightarrow \infty, p(\epsilon) \rightarrow \mathcal{P} \epsilon^{-1}$ and $d(\epsilon) \rightarrow \pi \delta(\epsilon)$, where $\mathcal{P}$ denotes principal value. Note the $d(\epsilon)$ controls the way energy is transferred. 


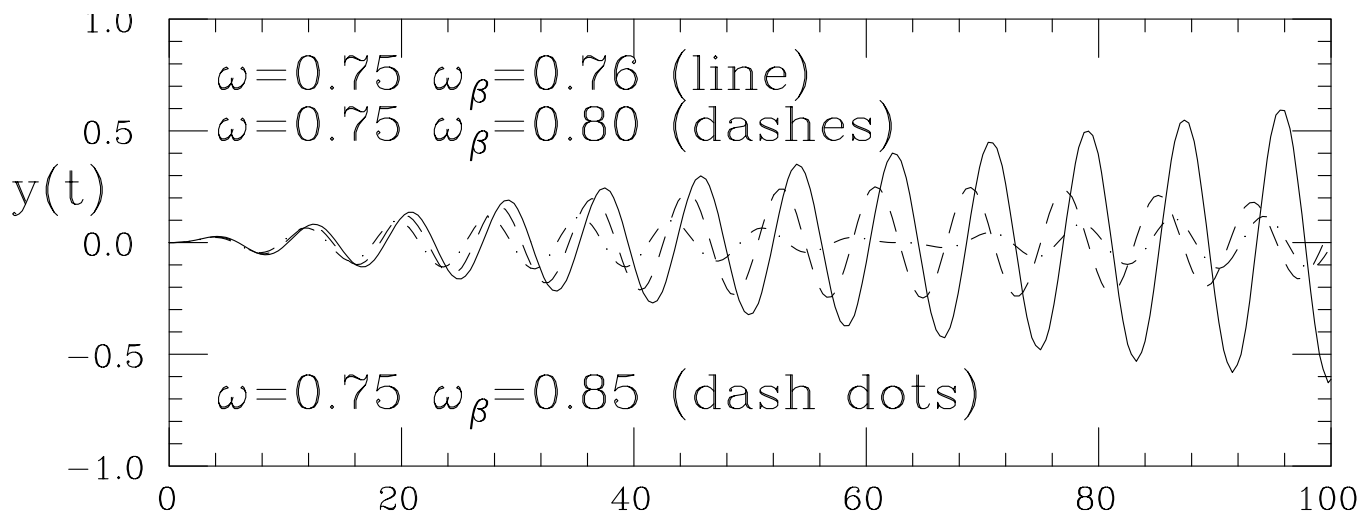

Figure 14: With a driving frequency of $\omega=0.75 \mathrm{~Hz}$, a particle with betatron frequency $\omega_{\beta}=$ $0.85 \mathrm{~Hz}$ (dash-dots), or $\epsilon=0.1 \mathrm{~Hz}$, is driven for $t \sim \pi /|\epsilon|=31$ s only before its amplitude decreases and oscillation energy is transferred to other particles. A particle with betatron frequency $\omega_{\beta}=0.80 \mathrm{~Hz}$ (dashes), or $\epsilon=0.05 \mathrm{~Hz}$, is driven for $t \sim \pi /|\epsilon|=63 \mathrm{~s}$ before its oscillation energy is given up. On the other hand, a particle with betatron frequency $\omega_{\beta}=0.76 \mathrm{~Hz}$ (dashes), or $\epsilon=0.01 \mathrm{~Hz}$, continues to absorb energy with its oscillation amplitude increasing linearly in the $100 \mathrm{~s}$ interval shown in the plot. It will give up its energy only after $t \sim \pi /|\epsilon|=314 \mathrm{~s}$.

$\omega_{\beta}=0.85$ (dash-dots), 0.80 (dashes), and $0.76 \mathrm{~Hz}$ (line) are displayed. The horizontal time scale is in seconds.

The energy absorbed by a beam particle from the driving force in time $t$ can be easily computed:

$$
E(t)=\int_{0}^{t} \dot{y} \hat{F} \sin \omega t^{\prime} d t^{\prime}=\frac{1}{2} \hat{F}^{2} \frac{\sin ^{2} \frac{1}{2} \epsilon t}{\epsilon^{2}}+\cdots,
$$

where only the leading term is shown. This again demonstrates that the particle first absorbs energy from the driving force and starts surrendering it back when $t \gtrsim \pi /|\epsilon|$. However, when its betatron frequency $\omega_{\beta}$ is extremely close to the driving frequency $\omega$, the absorption of energy continues. We also see that the total amount of energy imparted on a particle is bounded by $|\epsilon|^{-2}$. Thus more energy is absorbed by the particle if its betatron frequency is closer to the driving-force frequency. In fact, in the limit of $|\epsilon| \rightarrow 0$,

$$
E(t) \rightarrow \frac{1}{4} \hat{F}^{2} \pi t \delta(\epsilon) .
$$

If $\rho(\epsilon)$ denotes the betatron-frequency distribution of the beam particles, the total energy flowing into the beam becomes

$$
E(t) \rightarrow \frac{1}{4} \hat{F}^{2} \pi t \rho(0),
$$

which increases linearly. However, the energy is absorbed only by those few particles having betatron frequency exactly equal to the frequency of the driving force. As their oscillation 
amplitudes increase, these particles will eventually be lost to the walls of the beam pipe and the process of Landau damping stops.

In our discussion of Landau damping so far, the amplitude of the driving force $\hat{F}$ is independent of the system of particles. For an instability in a particle beam, the situation is slightly different. The driving force comes from the wakefields of the beam particles interacting with the discontinuities of the vacuum chamber, and usually has an amplitude proportional to the center displacement of the beam. When there is a kick to the beam that creates a center displacement $\langle y\rangle$ or a center displacement velocity $\langle\dot{y}\rangle$, a force with amplitude $\hat{F} \propto\langle y\rangle$ or $\langle\dot{y}\rangle$ is generated, which drives the whole ensemble of particles with the coherent frequency $\omega$. When $\omega$ is close to the slow wave frequency $\omega_{n, w_{0}}$ associated with some revolution harmonic $n$, slow waves with frequencies $\omega_{n, w}(\delta)$ corresponding to fractional momentum offset $\delta$ are excited, each of which will receive the amount of response according to

$$
\operatorname{amplitude}\left(\omega_{n, w}\right)=\frac{\hat{F}}{2 \bar{\omega}_{n, w}} \frac{\sin \left(\omega_{n, w}-\omega\right) t}{\omega_{n, w}-\omega},
$$

where $\bar{\omega}_{n, w}$ is the mean frequency of the system with a small half frequency spread $\Delta \omega_{n, w}$. Now two things happen. First, most excited slow waves give up their excited energy gradually to those slow waves having frequencies extremely close to $\omega$ through their coupling with the impedance. Second, the center of displacement of the beam starts to decay due to decoherence as a result of the betatron-frequency spread of the beam particles. As $\langle y\rangle$ decreases, the driving force decreases also. Finally the disturbance goes away. This is how Landau damping takes place in a beam. In fact, this process starts whenever the disturbance is of infinitesimal magnitude, implying that any disturbance will be damped as soon as it occurs. We say that there is enough Landau damping to keep the beam stable. Notice that no frictional force has ever been introduced in the whole process. Thus, there is still conservation of energy in the presence of Landau damping, which merely redistributes energy from waves of one frequency to another.

In case the half frequency spread $\Delta \omega_{n, w}$ is very narrow, it will take $t \approx \pi / \Delta \omega_{n, w}$ for the first wave to surrender its energy to another that has frequency closer to $\omega$. This time will be very long. Before this time arrives, all frequency components continue to receive energy and $\langle y\rangle$ continue to increase and so will the driving force. This is the picture of how an instability develops when the spread of frequency is not large enough to invoke Landau damping. The energy that feeds the instability may be extracted from the longitudinal energy of the beam resulting in a slower speed, or from the rf system that replenishes the beam energy. 


\section{LONGITUDINAL INSTABILITY OF A COASTING BEAM}

For a coasting beam, there is no rf voltage, and the unperturbed beam distribution $\Psi_{0}$ depends on only one variable $\delta$, the fractional momentum offset, and is normalized as $\int \Psi_{0} d \delta=1$. The beam generates wakefields which perturb the particle motion. The perturbed beam distribution $\Psi$ is a function of fractional momentum offset $\delta$ as well as time $t$ and azimuthal position $\theta$ around the ring. It obeys the Vlasov equation

$$
\frac{d \Psi}{d t}=\frac{\partial \Psi}{\partial t}+\dot{\theta} \frac{\partial \Psi}{\partial \theta}+\dot{\delta} \frac{\partial \Psi}{\partial \delta}=0
$$

where the overdot represents the derivative with respect to $t$. Let us assume the ansatz

$$
\Psi(\theta, \delta, t)=\Psi_{0}(\delta)+\Delta \Psi_{n} e^{j\left(\Omega_{c} t-n \theta\right)},
$$

where $n \neq 0$ because of charge conservation and $\Omega_{c}$ is the coherent frequency of the beam to be determined. This ansatz just states that the revolution harmonic number $n$ is a good eigennumber and its validity will be verified later. The perturbation represents a longitudinal harmonic wave with $n$ nodes and the effect of the impedance is the bunching of the coasting beam into $n$ microbunches.

The energy gained by a particle per revolution due to the wakefield is equal to the average current $I_{0}$ multiplied by the longitudinal impedance:

$$
\left.\Delta E\right|_{\text {per turn }}=-Z_{0}^{\|}\left(e I_{0} \int \Delta \Psi_{n} d \delta\right) e^{j\left(\Omega_{c} t-n \theta\right)},
$$

where the impedance is evaluated at the collective frequency $\Omega_{c}$. The negative sign on the right side signifies that the particle actually loses energy in the presence of a resistive impedance. The time derivative of the $\delta$ coordinate of the coasting beam becomes

$$
\dot{\delta}=-\frac{\omega_{0}}{2 \pi \beta^{2} E}\left(e I_{0} Z_{0}^{\|} \int \Delta \Psi_{n} d \delta\right) e^{j\left(\Omega_{c} t-n \theta\right)}
$$

where $E$ is the total energy of the beam particle. Since $\left|\Delta \Psi_{n}\right| \ll \Psi_{0}$ at the onset of collective instability, the Vlasov equation can be linearized to become

$$
j\left(\Omega_{c}-n \dot{\theta}\right) \Delta \Psi_{n}=\frac{\omega_{0} e I_{0} Z_{0}^{\|}}{2 \pi \beta^{2} E} \frac{d \Psi_{0}}{d \delta}\left(\int \Delta \Psi_{n} d \delta\right) .
$$

Moving $j\left(\Omega_{c}-n \dot{\theta}\right)$ to the right side, the last bracketed factor on the right side can be cancelled by an integration over $\delta$, and we obtain the dispersion relation

$$
1=-j \frac{e I_{0} \omega_{0} Z_{0}^{\|}}{2 \pi \beta^{2} E} \int \frac{d \Psi_{0} / d \delta}{\Omega_{c}-n \omega} d \delta
$$


where the revolution frequency $\dot{\theta}=\omega(\delta)$ is a function of momentum offset. The successful derivation of the dispersion relation implies that the ansatz given in Eq. (7.1) is selfconsistent.

Similar to the one for transverse instability, once the unperturbed distribution and the impedance are given, this dispersion relation is to be solved as an eigenequation to obtain the collective frequency $\Omega_{c}$, which is the eigenvalue, and the perturbed part of the distribution, which is the eigenfunction. If there is a solution with $\operatorname{Im} \Omega_{c}<0$, the amplitude of the $n$th harmonic wave grows exponentially and the beam encounters the collective microwave instability. The terminology is derived from the fact that the coherent frequency observed is in the microwave frequency range.

\subsection{No Spread in Frequency}

The dispersion relation in Eq. (7.6) can be integrated by part to become

$$
1=j \frac{e I_{0} n^{2} \omega_{0}\left(Z_{0}^{\|} / n\right)}{2 \pi \beta^{2} E} \int \frac{\Psi_{0}}{\left(\Omega_{c}-n \omega\right)^{2}} \frac{\partial \omega}{\partial \delta} d \delta .
$$

With the relation $\omega=\omega_{0}(1-\eta \delta)$ where $\eta$ is the frequency slip factor, the dispersion relation can be solved analytically for some distribution functions of the beam. Let us first consider the situation when there is no momentum spread among the beam particles. Then $\omega=\omega_{0}$ which is $\delta$ independent. The integral over $\delta$ is just the normalization of $\Psi$. We obtain

$$
\Omega_{c}=n \omega_{0} \pm \sqrt{-j \frac{e I_{0} n^{2} \omega_{0}^{2} \eta\left(Z_{0}^{\|} / n\right)}{2 \pi \beta^{2} E}} .
$$

In order that the beam is stable or $\operatorname{Im} \Omega_{c} \geq 0$, one must require $\eta Z_{0}^{\|} / n$ to be imaginary and positive. This implies that above/below transition a stable coasting beam must see a longitudinal impedance which is purely inductive/capacitive. This result is summarized in Table II.

Table II: Characteristic behavior of longitudinal collective instability without Landau damping.

\begin{tabular}{|l|l|l|l|l|}
\hline & $Z_{0}^{\|} / n$ & capacitive & inductive & resistive \\
\hline Below transition & $\eta<0$ & stable & unstable & unstable \\
\hline Above transition & $\eta>0$ & unstable & stable & unstable \\
\hline
\end{tabular}

The instability arises from the microbunching of the beam. Below transition, spacecharge impedance, which is capacitive, is repulsive and will therefore not be able to microbunch the beam. Above transition, however, the space-charge impedance, although it 
remains capacitive, becomes an attractive force. This explains why the beam can become unstable just after crossing transition if the impedance is dominated by space charge. The fact that a space-charge force becomes attractive is not conventional. One can envision such an attractive behavior if the mass of the particles becomes negative. As a result, microwave instability driven by the space-charge impedance above transition is often called negative-mass instability.

\subsection{Finite Momentum Spread}

With finite momentum spread among the beam particles, there exists finite region of the impedance space where there are no unstable solutions. Let us consider a Gaussian momentumoffset distribution for the unperturbed beam

$$
\Psi(\delta)=\frac{1}{\sqrt{2 \pi} \sigma_{\delta}} e^{-\delta^{2} /\left(2 \sigma_{\delta}^{2}\right)}
$$

where $\sigma_{\delta}$ is the rms spread. Substituting into Eq. (7.6) and letting $\omega(\delta)=\omega_{0}(1-\eta \delta)$, it is easy to obtain

$$
1=j \frac{e I_{0}\left(Z_{0}^{\|} / n\right)}{2 \pi \beta^{2} E \eta \sigma_{\delta}^{2}} \frac{1}{\sqrt{\pi}} \int_{-\infty}^{\infty} \frac{x e^{-x^{2}}}{x+z} d x,
$$

where we have defined the dimensionless quantities

$$
x=\frac{\delta}{\sqrt{2} \sigma_{\delta}} \quad \text { and } \quad z=\frac{\Omega_{c}-n \omega_{0}}{\sqrt{2} n \eta \omega_{0} \sigma_{\delta}} .
$$

Now the integral can be written easily in terms of the complex error function $w$. With the further substitution of

$$
U+j V=\frac{e I_{0}\left(Z_{0}^{\|} / n\right)}{2 \pi \beta^{2} E \eta \sigma_{\delta}^{2}},
$$

the dispersion relation takes the analytic form

$$
(j U-V)^{-1}=1+j z \sqrt{\pi} w(z) .
$$

Just as in the case of the transverse instability, contours of equal growth rates can be drawn in the $U-V$ space, where $U$ and $V$ are actually proportional to $\operatorname{sgn}(\eta) Z_{0}^{\|}$. Contours similar to this are shown in the left plot of Fig. 15. Unlike the situation when there is no momentum spread in the beam, we see clearly that the beam is stable in a finite impedance space. This is the result of Landau damping which counteracts the driving force of the impedance to a certain extent. Notice that these curves look bulb-shape and are different from those bell-shape curves in the transverse case in Fig. 12. The reason is obvious, here for the longitudinal we have the derivative of the unperturbed beam distribution $d \Psi_{0} / d \delta$ in the dispersion relation, whereas for the transverse we have the distribution $\rho(\delta)$. 


\subsection{KEIL-SCHNELL CRITERION}

For the dispersion relation of a coasting beam, we encounter the integral

$$
\int \frac{\Psi_{0}^{\prime}(x)}{x-z} d x
$$

where $z \propto\left(\Omega_{c}-n \omega_{0}\right)$, the collective frequency. There is one frequency for which the integral is easy to perform, for virtually any momentum distribution. ${ }^{*}$ It is when $z=0+j 0-$, or a point at the threshold contour when there is no frequency shift. It is actually the intercept of the threshold contour with the $V$-axis. For a Gaussian distribution in energy spread, stability requires

$$
-\frac{I_{m} Z_{\|}}{n} \leq \frac{2 \pi \eta E \sigma_{E}^{2}}{e I_{0} \beta^{2}}
$$

Since this intercept is usually the closest point of the threshold contour from the origin of the $U-V$ space, we approximate the threshold contour by a circle passing through the point to arrive at the stability criterion

$$
\left|\frac{Z_{\|}}{n}\right| \leq \frac{2 \pi|\eta| E \sigma_{E}^{2}}{e I_{0} \beta^{2}}
$$

If we perform the same computation for the parabolic distribution, and draw the circle in the same way, the criterion becomes

$$
\left|\frac{Z_{\|}}{n}\right| \leq \frac{2 \pi|\eta| E \sigma_{E}^{2}}{e I_{0} \beta^{2}} F
$$

where $F=\frac{5}{3}=1.67$. A stability criterion is useful only if it does not depend strongly on the energy distribution, or, in other words, when the form factor $F$ is close to unity for all distributions. Obviously, this is not the case here. The reason is that the rms energy spread is not a good description of a distribution when the edges of the distribution play a more important role in the Landau damping mechanism. Because of this, we try to use instead the full-width-at-half-maximum (FWHM) or half-width-at-half-maximum (HWHM). Now we define

$$
U^{\prime}+j V^{\prime}=\frac{e I_{0} \beta^{2} Z_{\|} / n}{\eta E(\Delta E / E)_{\mathrm{FWHM}}^{2}}
$$

and the dispersion relation now reads

$$
1=j\left(U^{\prime}+j V^{\prime}\right) \frac{2}{\pi} \int \frac{f^{\prime}(x) d x}{z-x},
$$

*There is also one such point in the transverse case. See Fig. 12. A circle can also be drawn through that point to obtain a Keil-Schnell transverse stability criterion. As will be demonstrated below, one should use the full-width-at-half-maximum spread rather than the rms spread. 
where the reduced collective frequency is denoted by $z$, the reduced energy distribution $f(x)$ is normalized to unity, and the spread of the distribution is determined by $x_{\text {ншнм }}=1$. The Keil-Schnell criterion [6] is now written as

$$
\left|\frac{Z_{\|}}{n}\right| \leq \frac{|\eta| E}{e I_{0} \beta^{2}}\left(\frac{\Delta E}{E}\right)_{\text {FWнм }}^{2} F
$$

where the form factor $F=\pi / 3=1.05$ for the parabolic distribution $f(x)=\frac{3}{4}\left(1-x^{2}\right)$ and $F=\pi /(4 \ln 2)=1.14$ for the Gaussian distribution $\frac{1}{\sqrt{2 \pi}} e^{-x^{2} / 2}$. For the tri-elliptic distribution $\frac{8}{3 \pi}\left(1-x^{2}\right)^{3 / 2}$, the bi-parabolic distribution $\frac{15}{16}\left(1-x^{2}\right)^{2}$, the quad-parabolic distribution $\frac{315}{32}(1-$ $\left.x^{2}\right)^{4}, F=1.06,1.07,1.10$, respectively. The criterion is more useful now because $F$ is in fact close to unity for quite a wide range of distributions.
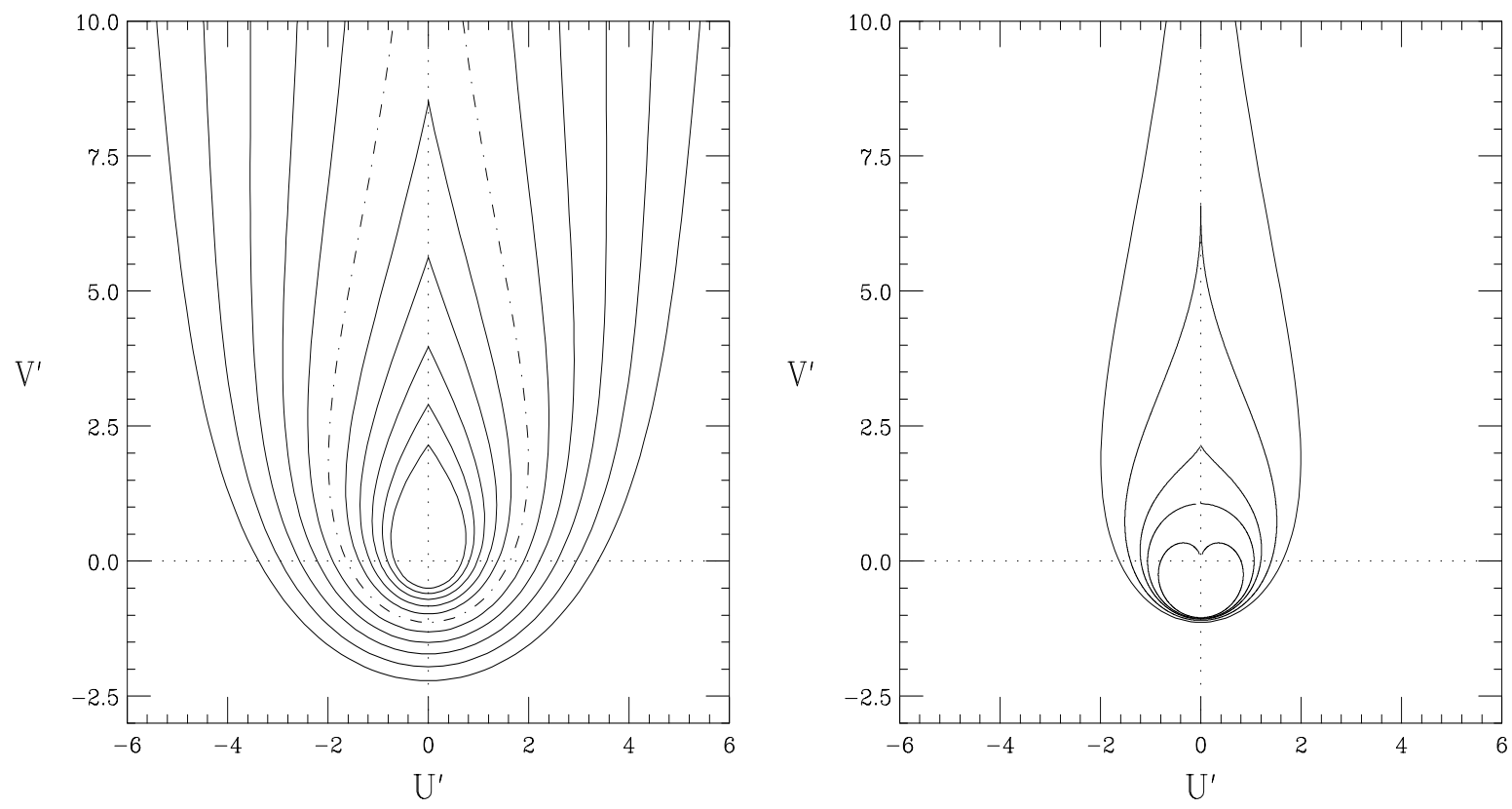

Figure 15: Left: The growth contours for a Gaussian distribution in revolution frequency below and above transition. The abscissa $U^{\prime}$ and ordinate $V^{\prime}$ are, respectively, real and imaginary parts of $e I_{0} \beta^{2}\left(Z_{0}^{\|} / n\right) /\left[\eta E(\Delta E / E)_{\mathrm{FWHM}}^{2}\right]$. From outside to inside, the contours correspond to growth rates 0.5 to -0.5 in steps of -0.1 in units of HWHM of the frequency spread, where negative values imply damping. The contour corresponding to the stability threshold is drawn in dot-dashes and the area inside it is stable. Right: The stability contours for different frequency distribution below and above transition. From inside to outside, they correspond to unperturbed revolution frequency distribution $f(x)=\frac{3}{4}\left(1-x^{2}\right), \frac{8}{3 \pi}\left(1-x^{2}\right)^{3 / 2}, \frac{15}{16}\left(1-x^{2}\right)^{2}, \frac{315}{32}\left(1-x^{2}\right)^{4}$, and $\frac{1}{\sqrt{2 \pi}} e^{-x^{2} / 2}$. Note that all threshold contours cut the $V^{\prime}$-axis at about -1 . 


\subsection{Boussard Conjecture}

Keil-Schnell stability criterion was derived for a coasting beam. Boussard [7] conjectured that the criterion should apply to a bunch also when the disturbance is much shorter than the length of the bunch and when the local current is used. Also the growth rate should be must faster than synchrotron frequency so that synchrotron oscillation can be neglected. Mathematically, applicability requires

(1) the average beam current $I_{0}$ replaced by local peak current $\hat{I}$,

(2) the wavelength of the broadband impedance much shorter than length of the bunch.

Thus the Boussard-modified Keil-Schnell stability criterion becomes

$$
\left|\frac{Z_{\|}}{n}\right| \leq \frac{|\eta| E}{e \hat{I} \beta^{2}}\left(\frac{\Delta E}{E}\right)_{\text {FWнм }}^{2} F .
$$

This postulate has been well-tested in the CERN Intersecting Storage Ring (ISR).

Krinsky and Wang [8] gave a vigorous derivation of the stability limit of a bunch with Gaussian energy distribution under the assumption that the wavelength of the perturbation is much shorter than the length of the bunch. Their result is exactly Eq. (7.16) with the average current $I_{0}$ replaced by the peak current $\hat{I}$. This derivation reconfirms Boussard conjecture.

\subsection{Observation}

In order for a bunch to be microwave unstable, the growth rate has to be much faster than the synchrotron frequency. For the Fermilab Main Ring, the synchrotron period was typically about 100 to 200 turns or 2 to $4 \mathrm{~ms}$. A naive way to observe the microwave growth is to view the spectrum of the bunch over a large range of frequencies at a certain moment and watch for the growth of some spectral region. However, the bunch spectrum produced by a network analyzer is usually via a series of frequency filters of narrow width, starting from low frequencies and working its way towards high frequencies. This process is time consuming. As soon as the filtering reaches the frequencies concerned, typically a few $\mathrm{GHz}$, the microwave growth may have been stabilized already through bunch dilution, and therefore no growth signals will be recorded. The correct way is to set the network analyzer at a narrow frequency span and look at the beam signal as a function of time. The frequency span is next set to an adjacent narrow frequency interval and the observation repeated until the frequency range of a few $\mathrm{GHz}$ has been covered. Besides, we must make sure that the network analyzer is capable of covering the high frequency of a few $\mathrm{GHz}$ for the microwave growth signals. The cable from the beam detector to the network analyzer must also be thick enough so that high-frequency attenuation is not a problem in signal 
propagation. Such an observation was made at the CERN Intersecting Storage Ring (ISR) which is a coasting beam machine. The network analyzer was set at zero span at $0.3 \mathrm{GHz}$. The beam current was at $55 \mathrm{~mA}$. The signal observed from injection for $0.2 \mathrm{~s}$ is shown at the lower left corner of Fig. 16 in a linear scale. We see the signal rise sharply and decay very fast, implying an instability which saturates very soon. The beam current was next increased by steps to $190 \mathrm{~mA}$ and the observation repeated. We notice that with a higher beam current, the instability starts sooner and stays on longer. The center frequency of the network analyzer was next increased at the steps of $0.2 \mathrm{GHz}$ and the observation repeated. The observation reveals an instability driven by a broadband impedance centering roughly at $1.2 \mathrm{GHz}$. Microwave instability can also be revealed in monitoring the longitudinal beam profiles, sometimes known as mountain ranges, via a wall resistance monitor. From the ripples, the frequency of the driving impedance can be determined. An example is shown in Fig. 17 for two consecutive turns of a chopped coasting beam at the Los Alamos Proton Synchrotron Ring (PSR). The source of the instability is a $\sim 72 \mathrm{MHz}$ resonance in a ferrite loaded pill box, designed to cancel the large space-charge impedance of the vacuum chamber. Unfortunately, the ferrite resistive impedance has been too large resulting in the the instability.

\section{BUNCH LENGTHENING}

When the current is above the microwave instability threshold, the energy spread of the beam may not just increase until the stability condition is restored. For protons and ions, measurements show that the energy spread increases to more than the threshold value. This phenomenon is called overshoot. An experiment observation gives approximately [9]

$$
\Delta E_{\text {before }} \Delta E_{\text {after }} \approx\left(\Delta E_{\text {threshold }}\right)^{2} .
$$

An electron bunch is quite different. Due to synchrotron radiation damping, there is no overshoot and the energy spread and bunch length just increase to the point at which the threshold condition is satisfied. We can therefore obtain an expression for the bunch length as a function of the impedance. In terms of the rms bunch length in azimuthal phase $\sigma_{\theta}=\omega_{0} \sigma_{\tau}$, the peak current of the Gaussian-distributed bunch is

$$
\hat{I}=\frac{e N_{B}}{\sqrt{2 \pi} \sigma_{\tau}}=\frac{\sqrt{2 \pi} I_{0}}{\sigma_{\theta}},
$$

where $I_{0}=e N_{B} f_{0}$ is the average bunch current. The bunch length is related to the energy spread by

$$
\sigma_{\theta}=\frac{\eta \sigma_{E}}{\beta^{2} \nu_{s}}
$$




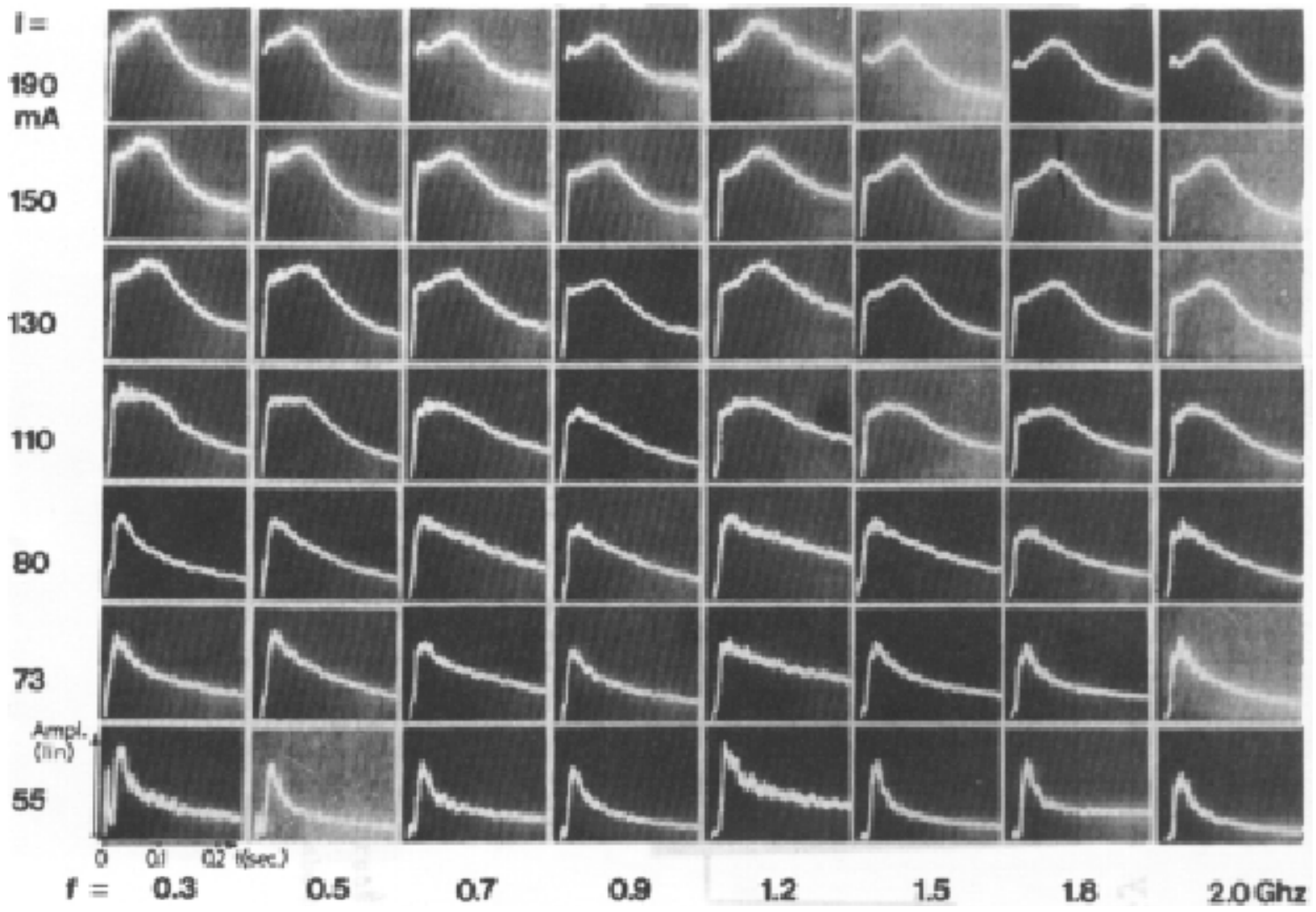

Figure 16: Pick-up signal after injection in the CERN ISR, for different observation frequencies but at zero span and different values of beam current. For high beam current, the signal grows before it decays.

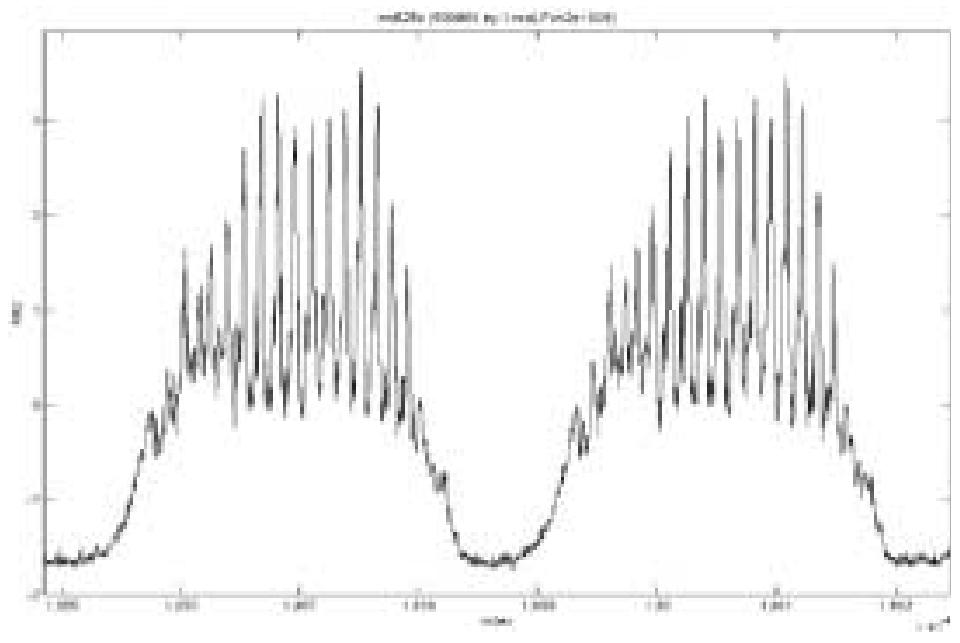

Figure 17: Beam profile of two consecutive turns of a chopped coasting beam recorded in a wallgap monitor after storage of $\sim 500 \mu \mathrm{s}$. The ripples show that a longitudinal microwave instability has occurred. 
Substituting into the Keil-Schnell threshold, we obtain

$$
\sigma_{\theta}=\left(\frac{e I_{0}\left|\eta Z_{0}^{\|} / n\right|}{\sqrt{2 \pi} F \beta^{2} \nu_{s}^{2} E}\right)^{1 / 3}
$$

Thus the bunch length depends only on the parameter

$$
\xi=\frac{e I_{0} \eta}{\beta^{2} \nu_{s}^{2} E}
$$

Chao and Garretye [10] showed that if the bunch samples the impedance with the frequency variation

$$
Z_{0}^{\|} \sim \omega^{a}
$$

the bunch length scales as

$$
\sigma_{\theta} \sim \xi^{1 /(2+a)}
$$

In order for Eq. (8.4) to satisfy the scaling law, we must identify $a=1$, or the impedance is a broadband. Actually, a broadband impedance was assumed when the dispersion relation was derived. The scaling law has been verified at many electron storage rings. The results for SPEAR at SLAC are shown in Fig. 18, where $\sigma_{\theta} \propto \xi^{0.78}$, giving $a=-0.68$. The behavior of $Z_{0}^{\|} \sim \omega^{-0.68}$ implies that the bunch is so short that it samples the region of the impedance at frequencies in the capacitive regime beyond the broadband peak.

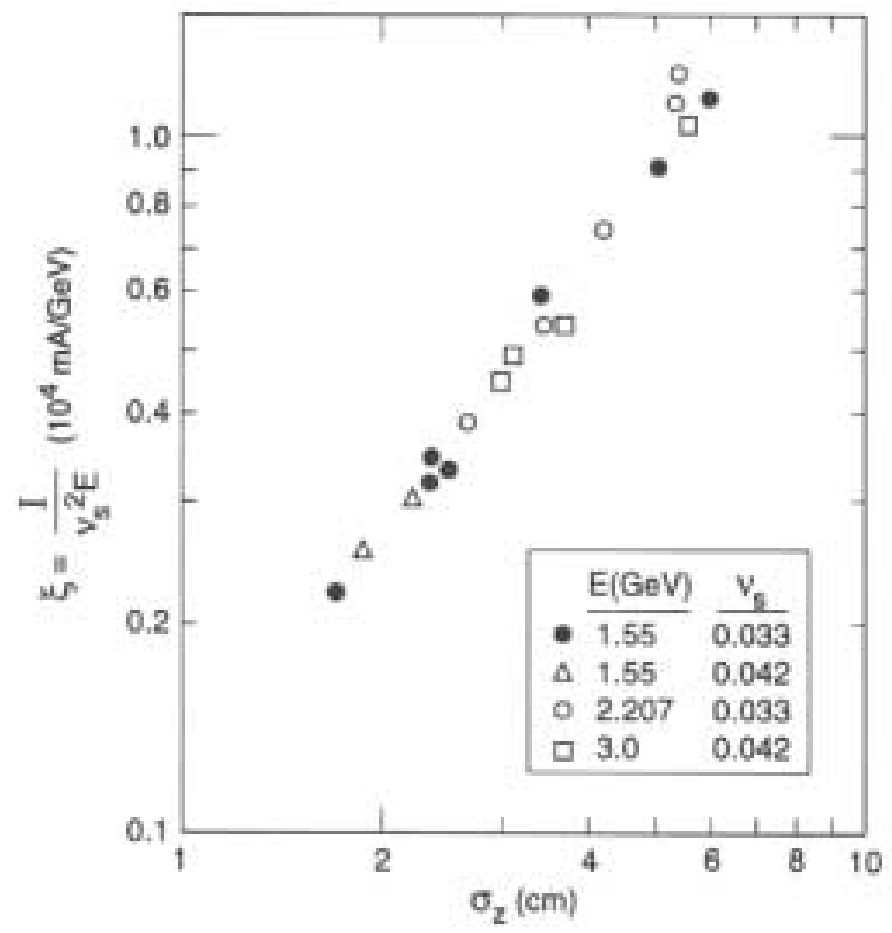

Figure 18: RMS bunch length $\sigma_{Z}$ versus the scaling parameter $\xi$ for the electron storage ring SPEAR. The momentum compaction factor has been kept constant. The measurement results indicate that $\sigma_{\tau} \propto \xi^{1 /(2+a)}$ with $a=-0.68$. 


\section{TRANSITION CROSSING}

We can rewrite Keil-Schnell stability criterion for a beam with Gaussian energy distribution by placing the growth rate without damping on the left side,

$$
\left(-\mathcal{I} m \Omega_{c}\right)_{\text {no damping }}=n \omega_{0} \sqrt{\frac{|\eta| e \hat{I}\left(Z_{0}^{\|} / n\right)}{2 \pi \beta^{2} E}} \leq n \omega_{0} \frac{|\eta| \sigma_{E}}{\beta^{2}}
$$

where the right side is the spread in frequency and can be interpreted as the rate of Landau damping.

Approaching transition $\eta$ becomes more and more less negative, reaches zero right at transition and increases positively afterwards. We see in above that as $|\eta| \rightarrow 0$, the damping rate goes to zero much faster than the growth rate. Thus there will be a time interval when the beam becomes unstable. If the machine is space-charge dominated near transition, the beam will be stable before transition and become unstable after crossing transition.

In the dispersion relation or the Keil-Schnell criterion, $\eta$ is the most rapid-varying variable and must be treated with care. But it is more than this. As $\eta$ is changing the bunch length $\sigma_{\tau}$ and the energy spread $\sigma_{E}$, and therefore the local peak current $\hat{I}$ are also changing, because they are functions of $\eta$ also. Near transition, we can write

$$
\eta=\frac{1}{\gamma_{T}^{2}}-\frac{1}{\gamma^{2}} \approx \frac{2 \dot{\gamma}_{T}}{\gamma_{T}^{3}} t
$$

where $\dot{\gamma}_{T}$ is the rate of change of $\gamma$ or the accelerating rate at transition, and $t$ is the time measured from the transition point. The dispersion relation is now solved with the substitution of this time-dependent slip factor, and the growth rate $\left(-\mathcal{I} m \Omega_{c}\right)_{\text {unstable }}$ is obtained as a function of time. The total growth across transition is then given by

$$
G=\exp \left\{\int_{\text {unstable }}\left(-\mathcal{I} m \Omega_{c}\right)_{\text {unstable }} d t\right\} .
$$

The bunch length and energy spread at each instant can be solved from the synchrotronmotion equation by assuming that the bunch area remains constant during the transition crossing. More involved consideration to include the potential distortion of the space-charge force can also be incorporated. Detailed derivation reveals that the bunch ellipse actually becomes tilted in the longitudinal phase space, because synchrotron oscillation is slowing down. All these consideration should be included in a credible computation. $[11,12]$

The next problem is the interpretation of the total growth computed in Eq. (9.3). This is not the growth of the bunch area. It is actually the growth of some spectral modes of the bunch that the impedance is perturbing. Let us assume that space charge is the main culprit. 
We learn before that $\mathcal{I}_{m} Z_{0}^{\|} / n$ is frequency independent. Thus, the growth rate, given by Eq. (9.1) increases linearly with frequency. The situation is actually not so bad because Im $Z_{0}^{\|} / n$ does roll off when the wavelength is of the order $a / \gamma$, where $a$ is the transverse size of the beam. Such a frequency dependency of the impedance is essential and must be included in the computation. The frequency of maximum growth is usually very large, for example $f_{\max } \sim 78 \mathrm{GHz}$ for the Fermilab Main Ring. We may ask what are the seeds of the growth at such high frequencies? Hardt's transition-crossing theory [13] says that the seeds come from the Schottky noise of the beam. We may wonder how can such a growth be important because the Schottky noise is so small. The answer is: the total growth can be enormous. Here we cite an example of the Fermilab Main Ring where the beam pipe radius $b=35 \mathrm{~mm}$ and beam radius $a=5 \mathrm{~mm}$. The synchronous phase was $60^{\circ}$ while crossing transition. Table III compares the final dimensionless power of the beam spectrum. Notice that if we choose the cutoff frequency, the seed coming from the beam linear distribution is big, but the total growth is small. On the other hand, if we choose $f_{\max } \sim 78 \mathrm{GHz}$, the seed coming from Schottky noise is small but the total growth is large. We see that when the original bunch area is $0.09 \mathrm{eV}-\mathrm{s}$ at $\dot{\gamma}_{T}=90 \mathrm{~s}^{-1}$ and total number of particles in the bunch $N_{b}=2.2 \times 10^{10}$, the power spectrum at cutoff frequency is 1.26 after crossing transition and is very much larger than the power spectrum of $1.41 \times 10^{-6}$ at $f_{\max }$, the maximum growth frequency. However, when the original bunch area is reduced to $0.05 \mathrm{eV}-\mathrm{s}$, while the power at cutoff grows to 3.70 only, the power at $f_{\max }$ grows to $1.5 \times 10^{9}$. Hardt also suggested a critical threshold parameter $c$; rapid growth will occur when $c>1$. Figure 19 shows the rapid growths of $c$ as functions of bunch area, respectively, for the Fermilab Main Ring and the Fermilab Main Injector.

Table III: Final fluctuation power spectra at cutoff and high-frequency Schottky harmonics.

\begin{tabular}{cccccc}
\hline$\dot{\gamma}_{t}$ & $N_{b}$ & Initial Bunch Emittance & \multicolumn{3}{c}{ Final Power Spectrum of Fluctuation } \\
$\left(\mathrm{s}^{-1}\right)$ & $\left(10^{10}\right)$ & $(\mathrm{eV}-\mathrm{s})$ & at $n_{\text {cutoff }}$ & at $n_{\text {max }}$ & sum \\
\hline 90 & 2.2 & 0.05 & 3.70 & $1.50 \times 10^{9}$ & $4.03 \times 10^{10}$ \\
90 & 2.2 & 0.06 & 2.21 & $1.08 \times 10^{2}$ & $3.97 \times 10^{3}$ \\
90 & 2.2 & 0.07 & 1.67 & $1.19 \times 10^{-2}$ & $5.74 \times 10^{-1}$ \\
90 & 2.2 & 0.08 & 1.41 & $4.86 \times 10^{-5}$ & $2.93 \times 10^{-3}$ \\
90 & 2.2 & 0.09 & 1.26 & $1.41 \times 10^{-6}$ & $1.06 \times 10^{-4}$ \\
\hline 120 & 4.0 & 0.06 & 7.44 & $4.37 \times 10^{18}$ & $1.00 \times 10^{20}$ \\
120 & 4.0 & 0.07 & 3.80 & $1.94 \times 10^{9}$ & $5.83 \times 10^{10}$ \\
120 & 4.0 & 0.08 & 2.54 & $4.40 \times 10^{3}$ & $1.67 \times 10^{5}$ \\
120 & 4.0 & 0.09 & 1.95 & $1.02 \times 10^{0}$ & $4.76 \times 10^{1}$ \\
120 & 4.0 & 0.10 & 1.64 & $3.57 \times 10^{-3}$ & $2.00 \times 10^{-1}$ \\
\hline
\end{tabular}


We learn from above that in order to reduce rapid growth during transition crossing, we can blowup the bunch area to a certain extent before crossing and/or increase the rate of acceleration near transition. A possible cure for microwave instability is to pass through the transition energy fast with a transition jump, which has been used successfully at the CERN PS. Numerical simulations provide a very valuable means of studying microwave blowup during transition crossing. However, the higher frequency components of the beam must be included in order to validate the simulations.
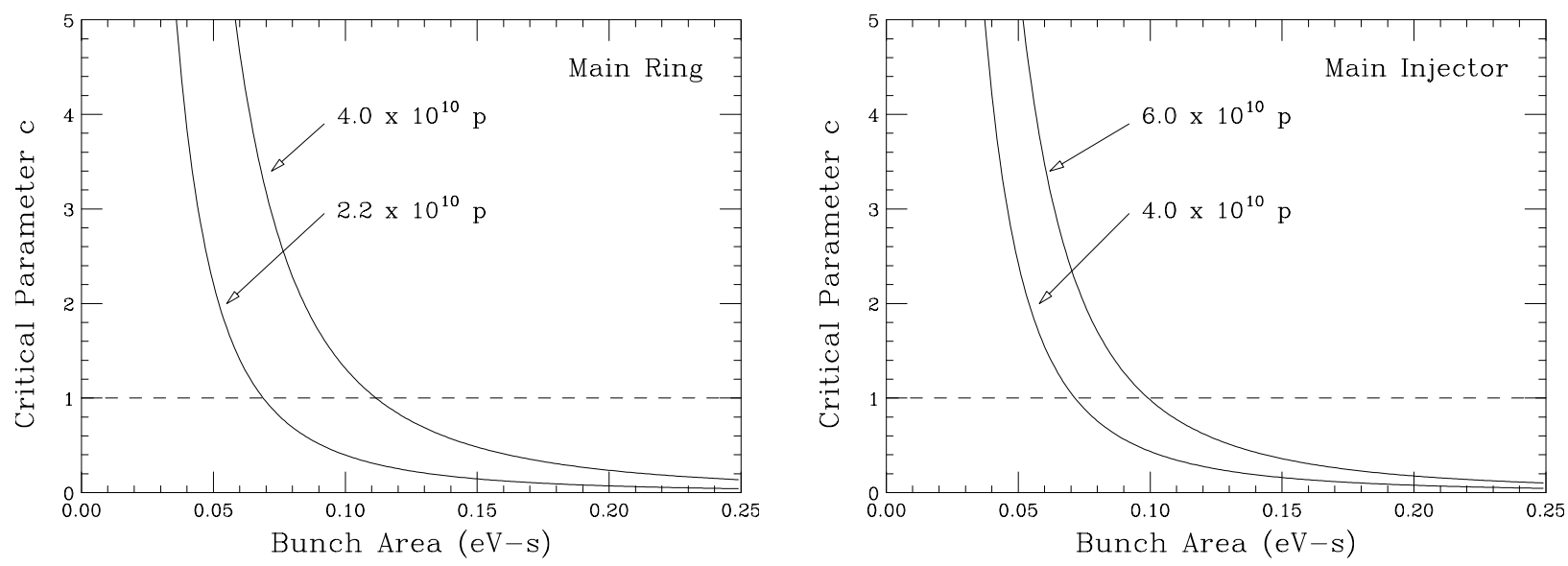

Figure 19: Plots showing the critical negative-mass parameter $c$ as a function of bunch area. Negative-mass blowup occurs when $c \gtrsim 1$. Left: the Fermilab Main Ring bunches with $N_{b}=$ $2.2 \times 10^{10}$ and $4.0 \times 10^{10}$ protons, while the ramp rate across transition is $\dot{\gamma}_{t}=90.0 \mathrm{~s}^{-1}$. Right: the Fermilab Main Injector bunches with $N_{b}=4.0 \times 10^{10}$ and $6.0 \times 10^{10}$ protons, while the ramp rate across transition is $\dot{\gamma}_{t}=160.1 \mathrm{~s}^{-1}$.

\section{Mode Coupling}

The dispersion relations we studied in the previous sections for transverse and longitudinal instabilities are designed for a coasting beam, where the revolution harmonic $n$ is a good eigennumber classifying the perturbed distributions. They may still be applicable to a bunch which is much longer than the wavelength of the driving force. A short bunch beam, on the other hand, cannot be described by the revolution harmonic, because synchrotron oscillation can no longer be neglected. Now the azimuthal mode number $m$ provides a good description of the collective bunch motion:* mode $m=1$ describes a slightly off-center bunch with the unperturbed bunch subtracted, mode $m=2$ describes the perturbed motion

*There should also be a radial eigennumber $n_{r}$, which we neglect here, denoting the number of nodes radially. 
of a bunch slightly mismatched to the rf bucket with the uperturbed bunch subtracted, etc. These are the synchrotron sidebands depicted in Fig. 8 when the bunch is small and when the perturbative impedance is small.

The dipole betatron oscillation of a small bunch is described by betatron sidebands depicted in Fig. 6. On top of the betatron motion, the bunch can also perform synchrotron motion in the longitudinal phase space. This gives rise to synchrotron sidebands on both sides of the synchrotron lines and the collective motion of the bunch motion. Thus the different collective transverse motions of the bunch are also classified by azimuthal mode number $m$. For example, mode $m=0$ is the rigid dipole mode where all beam particles are oscillating transversely in phase, mode ${ }^{\dagger} m= \pm 1$ describes head and tail oscillating differently leaving a node at the center of the linear density, and in general mode $m$ has $m$ nodes along the linear density.

When the beam intensity is small, the effect of wakefields on the bunch particles is small. These azimuthal modes are all separated by the synchrotron frequency $\omega_{s}$. As the beam intensity increases, the effect of wakefields become more evident. When the whole bunch has an offset from the axis of the beam pipe, the bunch is attracted further towards the wall of the beam pipe by its image charges, and its betatron frequency therefore becomes smaller. Thus, we expect to see the rigid dipole mode $m=0$ to decrease with increasing intensity while all other modes are not much affected. Thus, for small impedance, the azimuthal mode number $m$ remains a good eigennumber and the bunch is stable. When the bunch intensity is so strong that the $m=0$ has its frequency decreased by $\omega_{s}$, the two modes $m=0$ and -1 merge together as illustrated in the air-bag model in the left plot of Fig. 20. At this moment, the azimuthal mode number $m$ will not be able to describe the new bunch motion. Two new modes may result ${ }^{\ddagger}$ with complex frequencies: one is stable and the other unstable. This the so-called transverse mode-coupling instability, which was first observed at the DESY PETRA and later also at the SLAC PEP and the CERN LEP. It is also known as the strong head-tail instability, and is one of the cleanest instabilities to observe in electron storage rings, but has never been observed in hadron rings.

An estimate of the stability threshold can be obtained by requiring that the dipole coherent betatron frequency shift is less than $\omega_{s}$, or

$$
\left|Z_{1}^{\perp}\right|_{\mathrm{eff}} \lesssim \frac{2 E \omega_{\beta} \omega_{s} \tau_{L}}{e I_{0} c}
$$

where $\tau_{L}$ is the full bunch length, $I_{0}$ is the average bunch current, and the effective transverse

\footnotetext{
${ }^{\dagger}$ We need both the upper and lower synchrotron sidebands here because they are flanking the betatron lines and not the revolution harmonics. The symmetry about zero frequency is lost.

${ }^{\ddagger} \operatorname{Re} Z_{1}^{\perp}$ is required for the two modes to merge and become complex. If there $\operatorname{Re} Z_{1}^{\perp}=0$, the two modes just cross each other and the beam remains stable.
} 

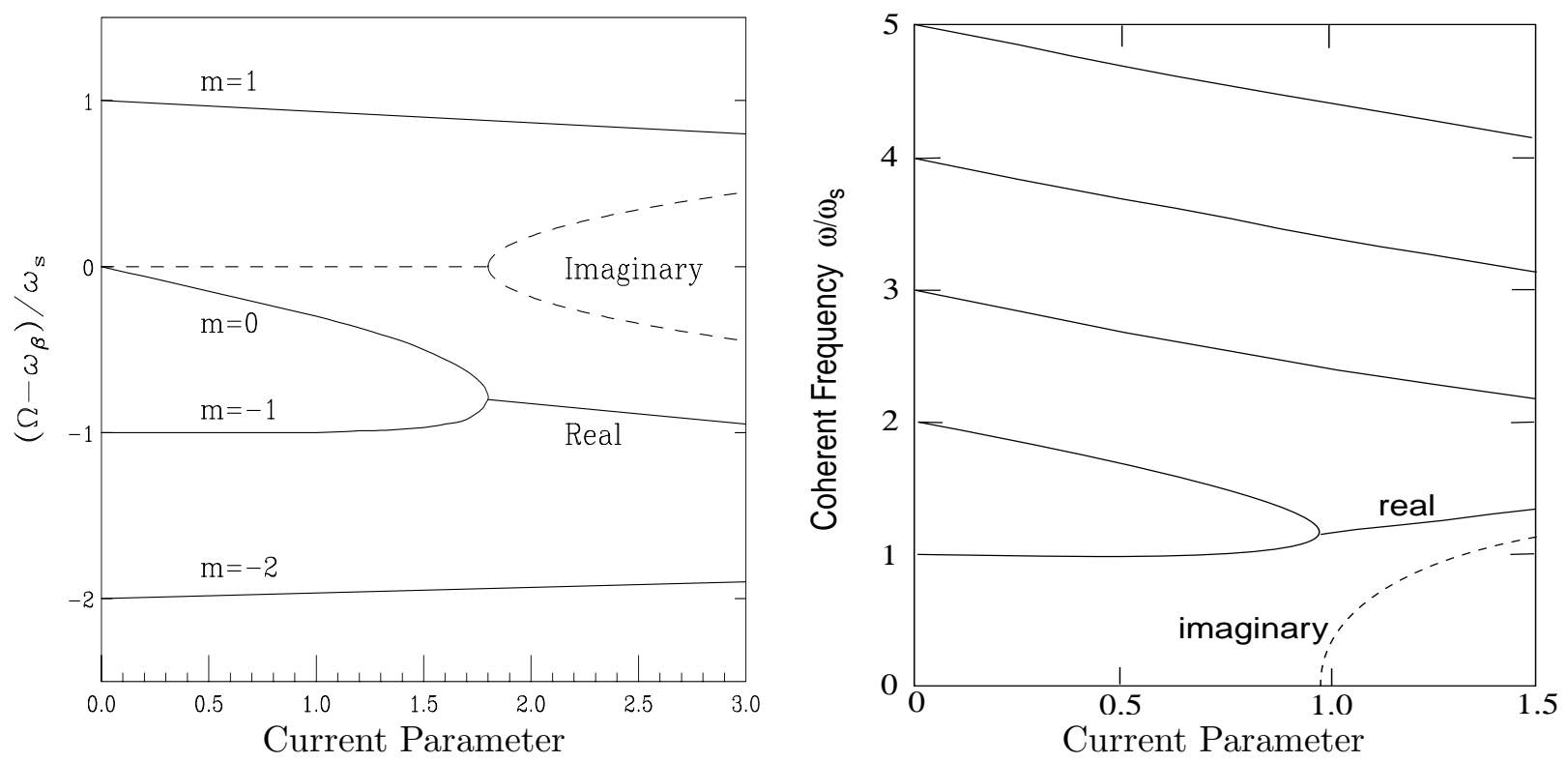

Figure 20: Left: Transverse mode frequencies $\left(\omega-\omega_{\beta}\right) / \omega_{s}$ versus the current parameter for an airbag bunch distribution. Right: Longitudinal mode frequencies $\omega / \omega_{s}$ versus the current parameter for a parabolic bunch distribution. The perturbation is a broadband impedance and the bunch is so short that all modes $m \geq 1$ sample the capacitive part of the impedance. The dashed curves are the imaginary part of the mode frequencies or growth/damping rates for the two colliding modes.

impedance is the transverse impedance averaged over the power spectrum $h(\omega)$ of the $m=0$ mode:

$$
\left|Z_{1}^{\perp}\right|_{\mathrm{eff}}=\frac{\int d \omega Z_{1}^{\perp}(\omega) h(\omega)}{\int d \omega h(\omega)} .
$$

Similar longitudinal mode-coupling instabilities are also possible. An illustration of an electron bunch in the parabolic distribution is shown in the right plot $^{\S}$ of Fig. 20. Here, the impedance is assumed to be broadband and the bunch is so short that all its azimuthal modes, with the possible exception of $m=1$, have frequencies higher than the broadband resonance peak. As a result, all the azimuthal modes, with the possible exception of $m=1$, sample the capacitive part of the impedance and have their frequencies shifted downward, remembering that the operation of the electron ring is above transition. Mode $m=1$ is a special mode, it depicts the rigid-bunch oscillation and is not affected by the reactive impedance, at least at low beam intensity. Physically, the center of the bunch does not see any change in the field pattern induced by the reactive impedance when the bunch motion is rigid. Mathematically, there is a static potential-distortion shift which cancels the dynamic shift. Thus, when the

\footnotetext{
${ }^{\S}$ Here we need to consider only upper synchrotron sidebands because the lower sidebands will lead to exactly the same physics as discussed earlier in Sec. 4.
} 
intensity of the bunch is low, all the azimuthal modes are separated by the synchrotron frequency $\omega_{s}$. As the beam intensity increases, mode $m=2$ is shifted downward while mode $m=1$ is essentially unchanged. When the beam intensity is high enough, these two modes merge to form two new states with complex frequencies and instability starts. ${ }^{\uparrow}$

If the bunch is long, like a proton bunch in the Fermilab Main Ring, because the wavelength of the perturbing broadband resonance is much shorter than the bunch length, some higher azimuthal modes, like $m=6$ and 7 in Fig. 21 will be the first to merge. Again

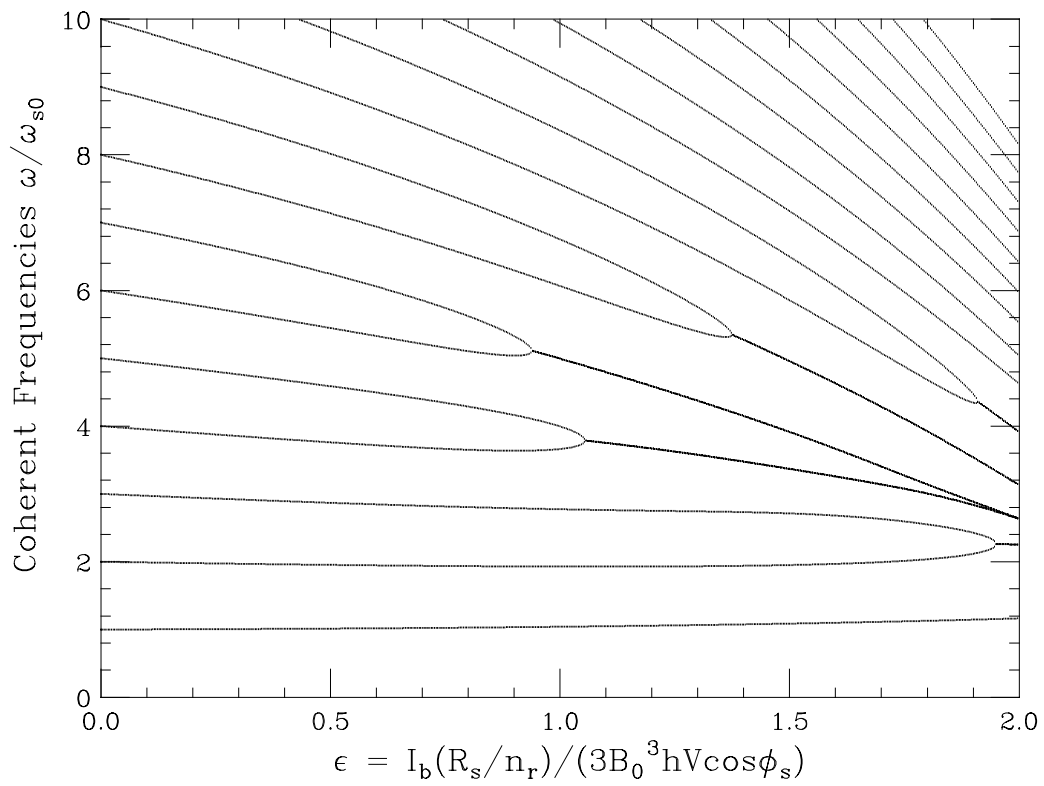

Figure 21: Longitudinal mode frequencies $\omega / \omega_{s}$ versus the current parameter for a long proton bunch of total length $\tau_{L}$ in the Fermilab Main Ring below transition. The impedance is a broadband resonance with $Q=1$ at $\omega_{r} /(2 \pi)=1.88 \mathrm{GHz}$, or $\omega_{r} \tau_{L} / \pi=7.5$. Since azimuthal modes $m=6$ and 7 peak at, respectively, $\omega_{m} \tau_{L} / \pi \approx 7$ and 8 , these two modes collide first. Since the width of the resonance encompasses modes 4 to 9 , collisions are seen for modes 4 and 5 as well as modes 8 and 9. For long bunches, usually many modes will collide after the first collision.

an estimate of the stability threshold can be obtained by requiring the coherent frequency shift of an azimuthal mode in the parabolic distribution to be less than $\omega_{s}$ :

$$
\left|\frac{Z_{0}^{\|}}{\omega}\right|_{\mathrm{eff}} \lesssim \frac{3 \beta^{2} E \omega_{s}^{2} \tau_{L}^{3}}{4 \pi^{2} e I_{b} \eta},
$$

\footnotetext{
`Nonzero $\operatorname{Re} Z_{0}^{\|}$is also required to produce complex frequencies.
} 
where the effective longitudinal impedance for mode $m$ is defined as

$$
\left|\frac{Z_{0}^{\|}}{\omega}\right|_{\mathrm{eff}}=\frac{\int d \omega \frac{Z_{0}^{\|}(\omega)}{\omega} h(\omega)}{\int d \omega h(\omega)} .
$$

It is very informative to understand the mechanism of the mode-coupling instabilities in the two-particle model. "[14] First, let us consider the situation when there is no external transverse focusing nor longitudinal focusing. If the head particle has a deviation from the axis of the beam pipe, it leaves a transverse wake so that the tail particle will be kicked transversely all the time. The result is a banana-tilted bunch with the tail curling sideway more and more until it hits the beam pipe. With external transverse focusing but without longitudinal focusing, a slightly off-axis head particle will perform betatron oscillation. The wake generated will force the tail to oscillate with the same betatron frequency with its amplitude increasing linearly until the tail hits the beam pipe. We see that the tail of the bunch will always be unstable transversely.

The picture becomes completely different when we turn on longitudinal focusing, because the particles exchange their head and tail positions. For example, particle 1 at the head will drive particle 2 at the tail with a linear rise in betatron amplitude for half a synchrotron period, during which particle 1 leads particle 2 in betatron phase by $90^{\circ}$. During the second half of the synchrotron period, particle 2 is at the head position and particle 1 is at the tail position. However, the phase relationship of the two particles has not been changed and particle 2 still lags particle 1 by $90^{\circ}$. Thus particle 2 is forced-damping particle 1 instead. If the wake is strong enough or the half synchrotron period is long enough, eventually the betatron amplitude of particle 1 will be damped to zero and start growing again with its betatron phase now lags that of particle 2 by $90^{\circ}$. Unlike a linac where the particle at the tail is driven to grow continuously by the particle at the head, here as the longitudinal positions of the two particles are exchanged, the new tail-particle will be damped to zero first before its amplitude is driven to grow. As a result, if the driving force from the wake is not large enough and/or the synchrotron period is not long enough, there can be no accumulation in amplitude growth and the two-particle bunch remains stable. A stability condition is such that both particles have the same betatron amplitudes $\hat{y}$ at the end of each half synchrotron period with the particle at the tail lagging the particle at the head by $90^{\circ}$. Since the driving force of the head particle needs to damp the tail particle from amplitude $\hat{y}$ to zero first and then drive it to $\hat{y}$ again, the total growth in amplitude, including the damping plus growing portions, for each half synchrotron period should be equal to $2 \hat{y}$.

\footnotetext{
"The bunch with $N_{B}$ particles is represented by two macroparticles each carrying charge $\frac{1}{2} e N_{B}$.
} 
By letting $\epsilon \rightarrow 0$ in Eq. (6.3), the amplitude of the driven tail particle after half a synchrotron period $t=\pi / \omega_{s}$ is

$$
\hat{y}_{t}=\frac{\hat{F}}{2 \omega_{\beta}} \frac{\pi}{\omega_{s}} .
$$

where from the definition of the transverse wake in Eq. (1.3) the wake force averaged over the circumference $C$ of the accelerator ring is

$$
\hat{F}=\frac{e^{2} N_{B} W_{0} c^{2}}{2 E C} \hat{y}_{h} .
$$

In above the charge of each macroparticle is $\frac{1}{2} e N_{B}$ where $N_{B}$ is the number of particles in the bunch, $E$ is the beam mean energy, $W_{1}=-W_{0}<0$ is the transverse wake experienced by the tail particle and is assumed constant for the length of the bunch. Since we require the total growth of the amplitude of the tail $\hat{y}_{t}$, the damped portion plus the growth portion, to be twice the amplitude of the head $\hat{y}_{h}$, we arrive at the stability condition

$$
\Upsilon=\frac{\pi e^{2} N_{B} W_{0} c^{2}}{4 E C \omega_{\beta} \omega_{s}} \leq 2
$$

which agrees very well with $\Upsilon \lesssim 1.8$ for the air-bag model shown in left plot of Fig. 20 . The result also agrees up to a factor of 2 with the estimate of Eq. (10.1), noting that in this constant-wake model $Z_{1}^{\perp}=-W_{0} /(\omega-j \epsilon)$ where $\epsilon$ is an infinitely small positive number and $\left.Z_{1}^{\perp}\right|_{\text {eff }}=j W_{0} h(0) / \int h(\omega) d \omega=j 4 W_{0} \tau_{L} / \pi^{2}$ because the normalization of the power spectrum $h(\omega)$.

\section{Head-Tail Instability}

We learn from the previous section that a bunch will not be unstable transversely in the presence of a broadband impedance when the beam current is not too large. The main reason is that, as the head and tail particles exchange positions, the new head mostly lags the new tail in betatron phase by $90^{\circ}$. Thus the tail will be damped for some time first before it starts to grow again. For this reason, there is a threshold for this strong head-tail instability, below which the beam is stable.

The above analysis is made when the chromaticity is zero. In the presence of a nonzero chromaticity, we will see below that under some condition the head of the bunch always leads the tail in betatron phase by less than $180^{\circ}$. Thus the bunch can become unstable without any threshold, aside from any damping mechanism like tune spread and mechanical damper.

Figure 22 shows a particle synchrotron motion in the longitudinal phase space below transition with positive chromaticity $(\xi>0)$. Starting at the head, the momentum offset 

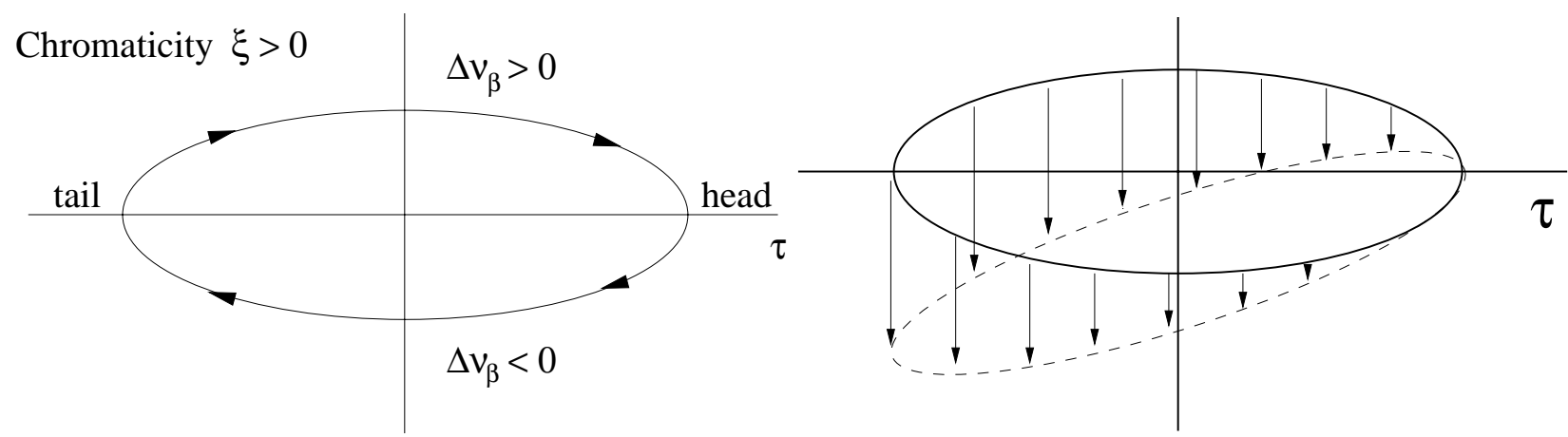

Figure 22: Schematic drawings showing betatron oscillation phase slipping from head to tail when chromaticity is positive $(\xi>0)$ and below transition $(\eta<0)$.

becomes negative, the particle betatron tune decreases, and its betatron phase $\phi_{\beta}$ slips. This slipping accumulates and reaches a maximum when the particle arrives at the tail. After that, because the momentum offset becomes positive, the betatron tune increases and $\phi_{\beta}$ regains its former slippage when it arrives at the head again.

Let us study this instability in the two-particle model. The first row of Fig. 23 shows the location of the two particles in longitudinal phase space below transition at start, $\frac{1}{4}$ synchrotron period, $\frac{1}{2}$ synchrotron period, and $\frac{3}{4}$ synchrotron period. The next two rows are for the $\sigma$-mode where the two particles are in phase at start. At $\frac{1}{4}$ synchrotron period, particle 1 (red) is at the head and particle 2 (green) at the tail. Because particle 1 has been at higher energy than particle 2, particle 1 now leads/lags particle 2 if the chromaticity is positive/negative. Thus, particle 1 can forced-drive/forced damped particle 2 . In the next $\frac{1}{4}$ synchrotron period, particle 2 slips forward/backward in betatron phase because it is at a higher energy while particle 1 slips backward/forward in betatron phase because its energy is less, so that they are again in phase at $\frac{1}{2}$ synchrotron period. In the next $\frac{1}{4}$ synchrotron period, particle 2 continues to slip forward/backward in betatron phase because it is at a higher energy while particle 1 continues to slip backward/forward in betatron phase because its energy is less. At $\frac{3}{4}$ synchrotron period, particle 2 reaches the head position while particle 1 reaches the tail position. Now particle 2 leads/lags particle 1 and can drive the amplitude of particle 1 to grow/damp. Thus this $\sigma$-mode is unstable/unstable below transition when the chromaticity is positive/negative.

There $\pi$-mode, where the two particles are $180^{\circ}$ out of phase at start, is treated in the lower part of Fig. 23. Following their motion in a synchrotron period, this mode is found stable/unstable below transition when the chromaticity is positive/negative. Above transition, because the particles move in the opposite direction in the longitudinal phase space, reverse conclusions are obtained. The results are summarized in Table IV. This is 


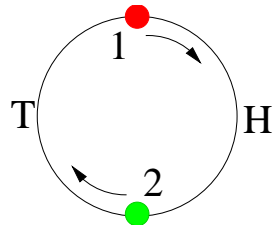

start

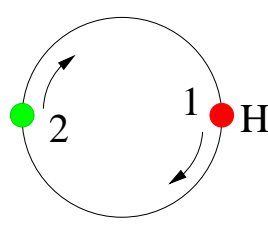

1/4 syn. period

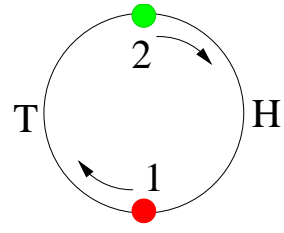

$1 / 2$ syn. period

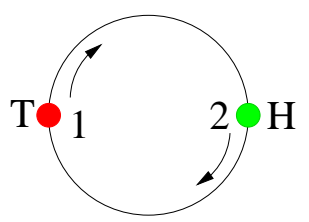

3/4 syn. period

\section{$\sigma$ mode}

Chromaticity $\xi>0$

$\longrightarrow \frac{1}{2}$

in phase

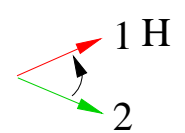

$\mathrm{H}$ leads $\mathrm{T}$ by $<180^{\circ}$

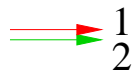

in phase

$\mathrm{H}$ leads $\mathrm{T}$ by $<180^{\circ}$

Unstable

Chromaticity $\xi<0$

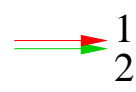

in phase

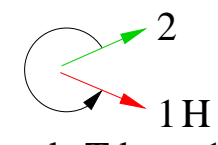

$\mathrm{H}$ leads $\mathrm{T}$ by $>180^{\circ}$

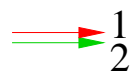

in phase

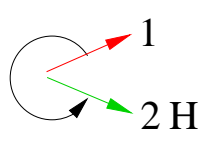

$\mathrm{H}$ leads $\mathrm{T}$ by $>180^{\circ} \quad$ Stable

\section{$\pi$ mode}

Chromaticity $\xi>0$

$2 \longrightarrow 1$

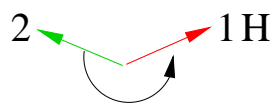

2

$180^{\circ}$ apart

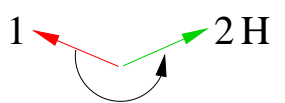

$\mathrm{H}$ leads $\mathrm{T}$ by $>180^{\circ}$
H leads T by $>180^{\circ} \quad 180^{\circ}$ apart
Stable

Chromaticity $\xi<0$

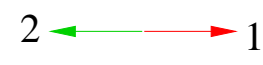

$2 \longrightarrow 1 \mathrm{H}$

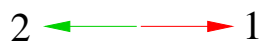

$180^{\circ}$ apart

$\mathrm{H}$ leads $\mathrm{T}$ by $<180^{\circ}$

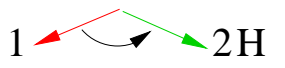

$\mathrm{H}$ leads $\mathrm{T}$ by $<180^{\circ}$

\section{Unstable}

Figure 23: (color) Analysis of chromaticity-driven head-tail instabilities below transition in the two-particle model. First row shows the positions and motion of the two particles (red for 1 and green for 2) in the longitudinal phase space. Below are the analysis of their betatron phase relationship in the $\sigma$ or rigid-dipole mode where the two particles are in phase, and the $\pi$-mode where the two particles are $180^{\circ}$ out of phase. 
Table IV: The chromaticity-driven head-tail stabilities and instabilities of the $\sigma$ - and $\pi$-modes in the two-particle model.

\begin{tabular}{|c|c|c|c|c|}
\hline & \multicolumn{2}{|c|}{$\sigma$-mode } & \multicolumn{2}{c|}{$\pi$-mode } \\
\cline { 2 - 5 } & $\xi>0$ & $\xi<0$ & $\xi>0$ & $\xi<0$ \\
\hline Below transition $\eta<0$ & unstable & stable & stable & unstable \\
Above transition $\eta>0$ & stable & unstable & unstable & stable \\
\hline
\end{tabular}

known as chromaticity-driven instability which occurs without a threshold. [15, 16]

In the two-particle model with a constant transverse wake, the growth or damping rate is given by

$$
\frac{1}{\tau}=\frac{e^{2} N_{B} W_{0} c^{2} \hat{\tau}}{2 \pi E C|\eta|},
$$

where $\hat{\tau}$ is the half bunch length. We see that under any condition, below or above transition, positive or negative chromaticity, the sum of the growth rates of the $\sigma$ - and $\pi$-modes is zero. Actually this sum rule remains true in a multiparticle bunch where there are many modes. In general, the growth or damping rate of the $\sigma$ or rigid-dipole mode is much larger than the growth or damping rates of all other higher modes.

It can be shown that, compared with the synchronous particle, the betatron phase lead or lag at a certain longitudinal position of the bunch is proportional to the distance in time $\tau$ from the center of the bunch. In fact,

$$
\Delta \phi_{\beta}=-\frac{\xi \omega_{0}}{\eta} \tau=-\omega_{\xi} \tau,
$$

where $\eta$ is the frequency slip factor. Thus this phase shift can be considered as a shift in the spectra of the bunch by the frequency $\omega_{\xi}$. The latter is called betatron-frequency-shift due to chromaticity.

Below/above transition, a negative/positive chromaticity implies shifting the bunch spectra to the right (positive frequency). We see in Fig. 24 that the rigid dipole mode $(m=0)$ samples the resistive-wall impedance more on the $\omega>0$ side than the $\omega<0$ side. Thus this mode is stable. However, the $m=1$ now samples more on the $\omega<0$ side than the $\omega>0$ side and becomes unstable. This is the so-called $\pi$-mode where the head and the tail are $180^{\circ}$ out of phase. To damp this mode a quadrupole damper is required. The higher modes (not shown) are also unstable. These higher modes have a number of nodes along the bunch and cannot be damped with a damper. However, the growth rates of the higher order modes are relatively slower. The usual operation employs a small negative chromaticity (positive above transition) so that the dominant dipole mode $(m=0)$ is stable. The higher modes, although unstable, will be Landau damped by a small spread in the betatron 


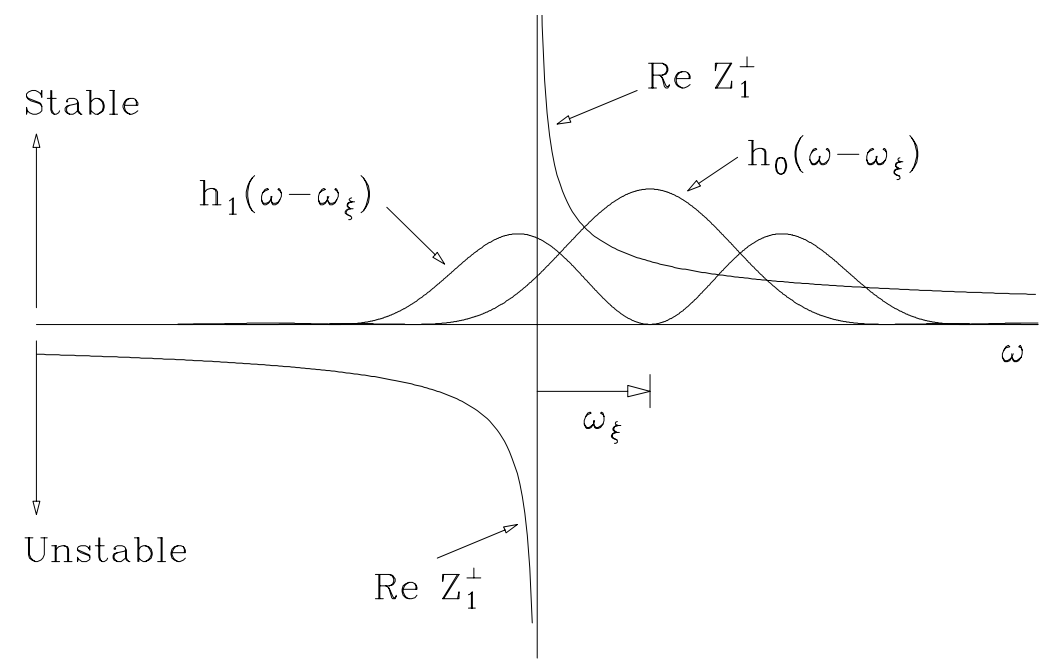

Figure 24: Negative/positive chromaticity below/above transition shifts the all modes of excitation towards the positive frequency side by $\omega_{\xi}$. Mode $m=0$ becomes stable, but mode $m=1$ may be unstable because it samples more negative $\operatorname{Re} Z_{1}^{\perp}$ than positive $\operatorname{Re} Z_{1}^{\perp}$.

tune. Another way is to employ a small positive chromaticity (negative above transition), so that the dipole mode $(m=0)$ becomes slightly unstable while all the higher order modes are stable. The rigid dipole mode is the one in which all particles are executing betatron oscillation in phase. This mode can easily be damped with a damper.

Head-tail modes of oscillations can be excited by shifting the chromaticity to the unstable direction and observed using a wideband pickup. These modes were first observed in the CERN PS Booster [17] and depicted in Fig. 25.

\section{COUPLED-BUNCH INSTABILTIES}

When the wake does not decay within the bunch spacing, bunches talk to each other. Assuming $M$ bunches of equal intensity equally spaced in the accelerator ring, there are $\mu=0,1, \cdots, M-1$ modes of oscillations in which the center-of-mass of a bunch leads* its predecessor by the phase $2 \pi \mu / M$. In addition, an individual bunch in the $\mu$ th coupledbunch mode can oscillate in the synchrotron phase space about its center-of-mass in the $m$ th azimuthal mode with $2 m=2,4, \cdots$ azimuthal nodes ${ }^{\dagger}$ in the perturbed longitudinal phase-space distribution. Of course, there will be in addition radial modes of oscillation in

${ }^{*}$ We can also formulate the problem by having the bunch lag its predecessor by the phase $2 \pi \mu^{\prime} / M$ in the $\mu^{\prime}$ th coupling mode. Then mode $\mu^{\prime}$ will be exactly the same as mode $M-\mu$ discussed in the text.

$\dagger$ For example, the dipole mode $m=1$ can be written as $\sim \cos \phi$, which has two nodes $\phi= \pm \pi / 2$. 

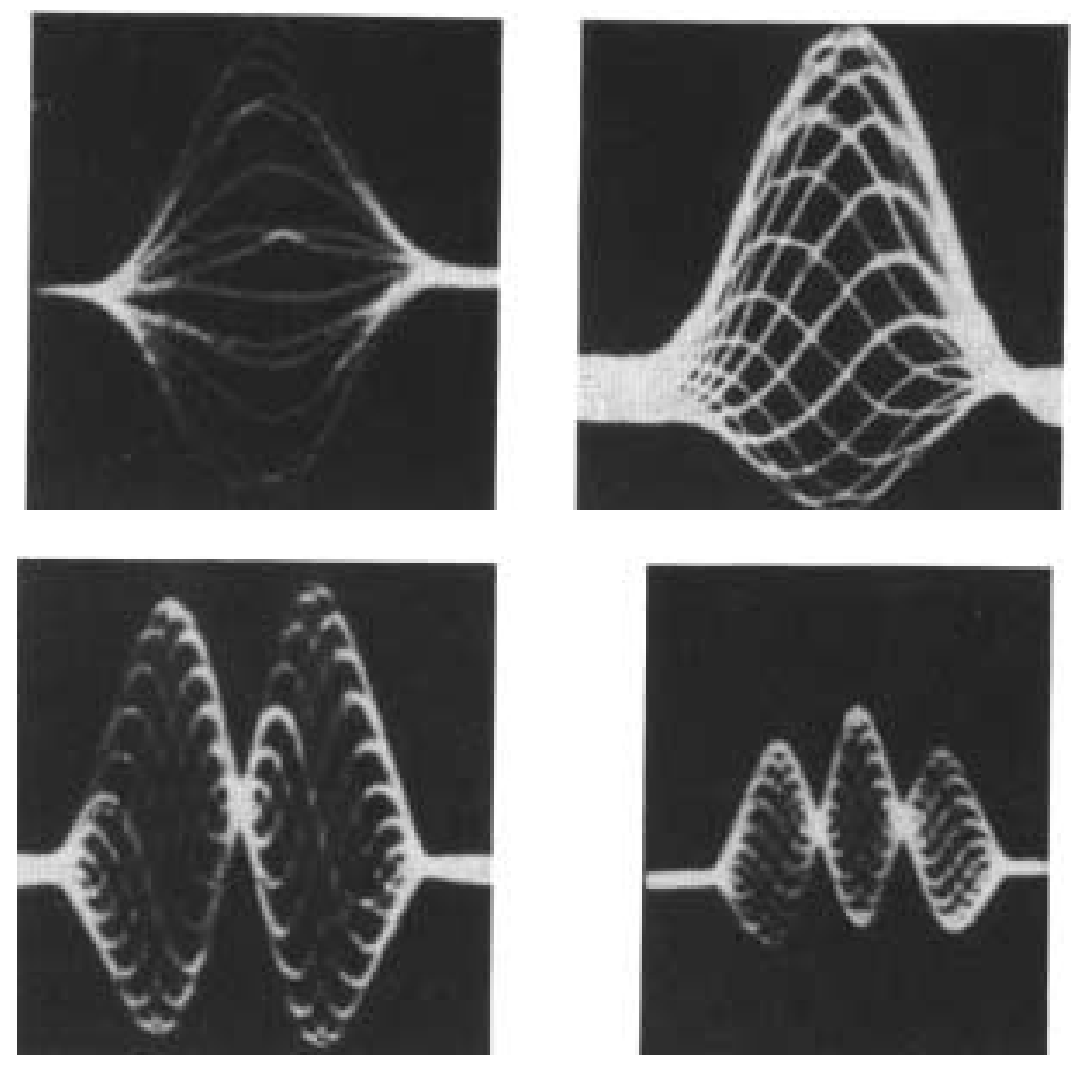

Figure 25: small A single bunch in the CERN PS Booster monitored in about 20 consecutive revolutions with a wideband pickup (bandwidth $\sim 150 \mathrm{MHz}$ ). Vertical axis: difference pickup signal. Horizontal axis: time (50 ns per division). The azimuthal mode number and chromaticity in each plot are as labeled.

the perturbed distribution.

The long-range longitudinal wake can drive longitudinal coupled-bunches instability, while the long-range transverse wake can drive transverse coupled-bunch instability. The source of the long-range driving force is usably sharp parasitic resonances in the rf cavities. However, the most serious transverse coupled-bunch instability that occurs in nearly all storage rings is the one driven by the resistive wall [18]. Since $\mathcal{R}^{\ddagger} Z_{1}^{\perp} \propto \omega^{-1 / 2}$ and is positive (negative) when the angular frequency $\omega$ is positive (negative), the betatron line at the lowest negative frequency acts like a narrow resonance and drives transverse coupledbunch instabilities. Take, for example, the Fermilab Tevatron in the fixed-target mode, where there are $M=1113$ equally spaced bunches. The betatron tune is $\nu_{\beta}=19.6$. The

\footnotetext{
${ }^{\ddagger}$ Here, we assume that the wall is thicker than one skin depth at revolution frequency. Otherwise, $\operatorname{Re} Z_{1}^{\perp} \propto \omega^{-1}$.
} 


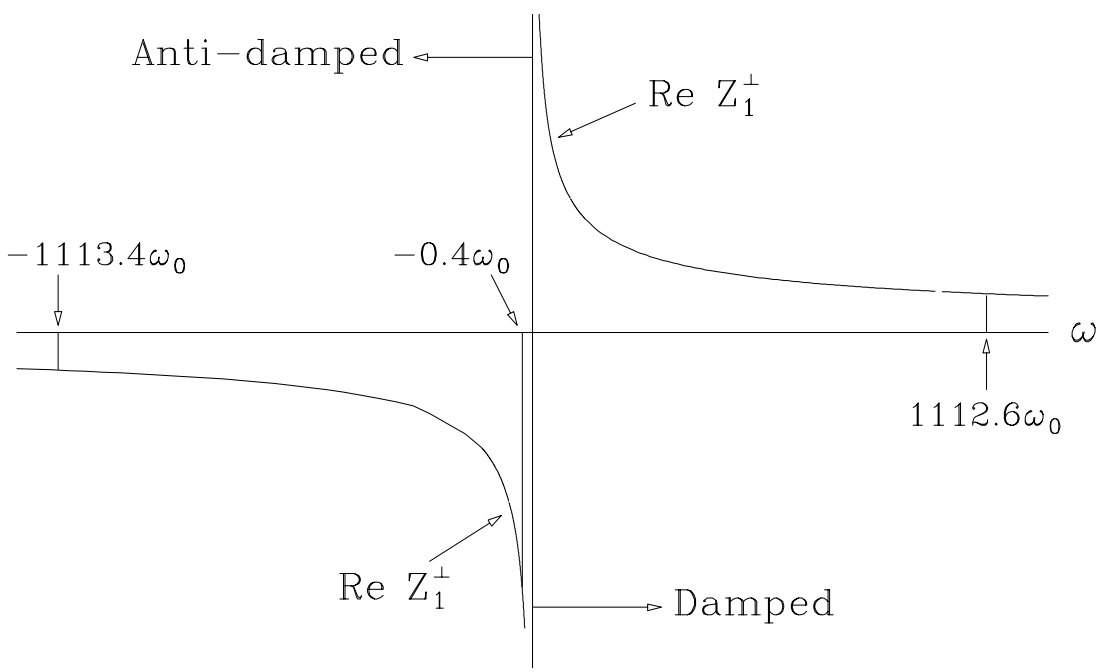

Figure 26: The $-0.4 \omega_{0}$ betatron line in the Tevatron dominates over all other betatron lines for the $\mu=1093$ mode coupled-bunch instability driven by the resistive wall impedance.

lowest-negative-betatron-frequency line is at $(q M+\mu) \omega_{0}+\omega_{\beta}=-0.4 \omega_{0}$, for mode $\mu=1093$ and $q=-1$. The closest damped betatron $\operatorname{line}^{\S}(q=0)$ is at $(1113-0.4) \omega_{0}$, but $\operatorname{Re} Z_{1}^{\perp}$ is only $-\sqrt{0.4 / 1112.6}$ the value at $-0.4 \omega_{0}$. The next anti-damped betatron line $(q=-2)$ is at $-1113.4 \omega_{0}$, with $\operatorname{Re} Z_{1}^{\perp}$ equal to $\sqrt{0.4 / 1113.4}$ the value at $-0.4 \omega_{0}$. This is illustrated in Fig. 26. Thus, it is the $-0.4 \omega_{0}$ betatron line that dominates. The growth rate for this mode can be written as

$$
\frac{1}{\tau_{m \mu}} \approx-\frac{1}{1+m} \frac{e M I_{b} c}{4 \pi \nu_{\beta} E_{0}} \mathcal{R} e Z_{1}^{\perp}\left(\omega_{q}\right) F_{m}^{\prime}\left(\omega_{q} \tau_{L}-\chi\right)
$$

where $\chi=\omega_{\xi} \tau_{L}$ and the form factor is

$$
F_{m}^{\prime}\left(\omega \tau_{L}\right)=\frac{2 \pi h_{m}(\omega)}{\tau_{L} \int_{-\infty}^{\infty} h_{m}(\omega) d \omega}
$$

where $h_{m}(\omega)$ is the power spectrum of the azimuthal mode $m$. For zero chromaticity, only the $m=0$ mode can be unstable because the power spectra for all the $m \neq 0$ modes are nearly zero near zero frequency. Since the perturbing betatron line is at extremely low frequency, we can evaluate the form factor at zero frequency. For the sinusoidal modes, we get $F_{0}^{\prime}(0)=8 / \pi^{2}=0.811$.

One method to make this coupled-bunch mode less unstable or even stable is by introducing positive chromaticity when the machine is above transition. For the Tevatron with

\footnotetext{
$\S$ When we have exact symmetry of $M$ bunches, for each mode, we do not have the betatron lines flanking each revolution harmonic. They only appear for every $M$ th harmonic.
} 
slip factor $\eta=0.0028$, total bunch length $\tau_{L}=5 \mathrm{~ns}$, and revolution frequency $f_{0}=47.7 \mathrm{kHz}$, a chromaticity of $\xi=+10$ will shift the spectra by the amount $\chi=\omega_{\xi} \tau_{L}=2 \pi f_{0} \xi \tau_{L} / \eta=5.4$. The form factor and thus the growth rate is reduced by more than 4 times. However, from Fig. 24 , the spectra are shifted by $\omega_{\xi} \tau_{L} / \pi=1.7$ and the $m=1$ mode becomes unstable. Another method for damping the instability is to introduce a betatron angular frequency spread using octupoles, with the spread larger than the growth rate. A third method is to employ a mode damper. One can also reduce the resistive-wall impedance by coating the walls of the beam pipe with a layer of copper, whose conductivity at cryogenic temperature is very much larger than the conductivity of stainless steel.

When all the $h=1113 \mathrm{rf}$ buckets are filled with $6 \times 10^{10}$ protons each in one scenario of the Tevatron in the fixed-target mode, the average total current is $M I_{b}=0.511 \mathrm{~A}$. The vertical resistive-wall impedance has a real part $\mathcal{R} e Z_{1}^{\perp}=43.74 \mathrm{M} \Omega / \mathrm{m}$ at the revolution harmonic. Thus, at $-0.4 \omega_{0}$, it becomes $\mathcal{R e} Z_{1}^{\perp}=-69.16 \mathrm{M} \Omega / \mathrm{m}$. At the injection energy of $E_{0}=150 \mathrm{GeV}$ and zero chromaticity, the transverse coupled-bunch growth rate driven by the resistive-wall impedance is $\tau_{\mu}^{-1}=232 \mathrm{~s}^{-1}$ and the growth time is $4.30 \mathrm{~ms}$ or 204 revolution turns. The mean radius of the Tevatron ring is $R=1 \mathrm{~km}$. In fact, this growth time is more or less the same for all accelerator rings [19]. For example, preceding the Tevatron, there are the Main Injector and the Booster. All of them have the same $53-\mathrm{MHz}$ rf. The Main Injector has $588 \mathrm{rf}$ buckets and the Booster has $84 \mathrm{rf}$ buckets. First, if all the buckets of each ring are filled, the average total current $M I_{b}$ should be the same for all the 3 rings. Second, the beam energy $E_{0}$ scales as the size of the ring or the mean radius $R$ and betatron tune $\nu_{\beta}$ scales as $\sqrt{R}$. Third, the resistive-wall impedance, as given by

$$
Z_{1}^{\perp}(\omega)=[1+j \operatorname{sgn}(\omega)] \frac{2 R c \rho}{\omega b^{3} \delta_{\text {skin }}}
$$

in Eq. (2.23), where $b$ is the beam pipe radius, $\delta_{\text {skin }}$ is the skin depth, and $\rho$ is the resistivity, scales as $R^{3 / 2}$ because the revolution frequency scales as $R^{-1}$. Substituting into Eq. (12.1), we find that the growth rate turns out to be independent of the size of the ring. Of course, usually there are differences in the vacuum chamber, and number of particles per bunch, and also the residual betatron tune. However, it is safe to say that the growth time of transverse couple-bunch instability for every completely filled accelerator ring should be of the order of a few to a few tens of milliseconds. Although the growth time is independent of the size of the ring, the growth time in turn number is inversely proportional to the size of the ring. Thus, for the Very Large Hadron Collider (VLHC) under consideration with a circumference of $233 \mathrm{~km}$, the growth time will be only 5.5 revolution turns according to this scaling and assuming the residual tune to be $\frac{1}{2}$. For this reason, large machines will require powerful feedback systems, for example, criss-crossing feedback and/or one-turn correction scheme.

There is no counterpart for the longitudinal resistive-wall impedance. This is because 
the amplitude of the $m$ th synchrotron sideband flanking the zero harmonic is, according to Eq. (4.3), proportional to $J_{m}\left(n \omega_{0} \hat{\tau}\right)$, which is exactly zero when $n=1$. (see Fig. 8).

\section{References}

[1] W.K.H. Panofsky and W.A. Wenzel, Rev. Sci. Instr. 27, 967 (1956).

[2] F. Sacherer, RMS Envelope Equations with Space Charge, IEEE Trans. Nucl. Sci. NS18, (PAC 1971) 1105 (1971). See also the longer report of the same title in CERN-SIInt.-DL/70-12, Nov., 1970.

[3] S. Machida and M. Ikegami, Simulation of Space Charge Effects in a Synchrotron, Proceedings of Workshop on Space Charge Physics in High Intensity Hadron Rings, p.73, Ed. Luccio, A.U., and Weng, W.T., (Shelter Island, New York, May 4-7, 1998).

[4] R. Baartman, Betatron Resonances with Space Charge, Proceedings of Int. Workshop on Emittance in Circular Accelerators Nov. 1994, KEK, Japan, KEK report 95-7, p.273; R. Baartman, Betatron Resonances with Space Charge, Proceedings of Workshop on Space Charge Physics in High Intensity Hadron Rings, p.73, Ed. Luccio, A.U., and Weng, W.T., (Shelter Island, New York, May 4-7, 1998).

[5] P.B. Robinson, Stability of Beam in Radiofrequency System, Cambridge Electron Accel. Report CEAL-1010, 1964.

[6] E. Keil and W. Schnell, CERN Report TH-RF/69-48 (1969); V.K. Neil and A.M. Sessler, Rev. Sci. Instr. 36, 429 (1965).

[7] D. Boussard, CERN Report Lab II/RF/Int./75-2 (1975).

[8] S. Krinsky and J.M. Wang, Particle Accelerators 17, 109 (1985).

[9] R.A. Dory, Thesis, MURA Report 654, 1962.

[10] A.W. Chao and J. Gareyte, Part. Accel. 25, 229 (1990).

[11] S.Y. Lee and J.M. Wang, Microwave Instability Across the Transition Energy, IEEE Trans. Nucl. Sc. NS-32, 2323 (1985).

[12] J. Wei, Longitudinal Dynamics of the Non-Adiabatic Regime on Alternating-Gradient Synchrotrons, PhD Dissertation, SUNY, Stony Brook, 1990.

[13] W. Hardt, Gamma-Transition-Jump Scheme of the CPS, Proc. 9th Int. Conf. High Energy Accel., SLAC, Stanford, 1974, p.434. 
[14] R. Talman, The Influence of Finite Synchrotron Oscillation Frequency on the Transverse Head-Tail Effect, Nucl. Instr. Meth. 193, 423 (1982).

[15] C. Pellegrini, Nuovo Cimento 64, 447 (1969).

[16] M. Sands, SLAC-TM-69-8 (1969).

[17] K. Hübner, P. Strolin, V. Vaccaro, and B. Zotter, Concerning the Stability of the ISR Beam Against Coherent Dipole Oscillations, CERN/ISR-RF-TH/70-2 (1970).

[18] F.J. Sacherer, Theoretical Aspects of the Behaviour of Beams in Accelerators and Storage Rings, Proc. First Course of Int. School of Part. Accel., Erice, Nov. 10-22, 1976, p.198.

[19] W. Chou and J. Griffin, Impedance Scaling and Impedance Control, Proceedings of the 1997 Particle Accelerator Conference, Vancouver, Canada, May 12-16, 1997, p. 1724. 